

\title{
A two-dimensional continued fraction algorithm with Lagrange and Dirichlet properties
}

\author{
par CHRISTIAN DROUIN
}

\begin{abstract}
RÉsumÉ. On démontre dans cet article un Théorème de Lagrange, pour un certain algorithme de fraction continue en dimension 2, dont la définition géométrique est très naturelle. Des propriétés type Dirichlet sont aussi obtenues pour la convergence de cet algorithme. Ces propriétés proviennent de caractéristiques géométriques de l'algorithme. Les relations entre ces différentes propriétés sont étudiées. En lien avec l'algorithme présenté, sont rapidement évoqués les travaux de divers auteurs dans le domaine des fractions continues multidimensionnelles.
\end{abstract}

ABstract. A Lagrange Theorem in dimension 2 is proved in this paper, for a particular two dimensional continued fraction algorithm, with a very natural geometrical definition. Dirichlet type properties for the convergence of this algorithm are also proved. These properties proceed from a geometrical quality of the algorithm. The links between all these properties are studied. In relation with this algorithm, some references are given to the works of various authors, in the domain of multidimensional continued fractions algorithms.

\section{Introduction and results}

1.1. Quick presentation of the main results. Since the beginning of the theory of Multidimensional Continued Fractions, an extension of the well known Lagrange Theorem in dimension one has been searched for. Historical remarks on the multidimensional continued fractions (the JacobiPerron algorithm and others) can be found in the works by F. Schweiger: [22] and [23], and A.J. Brentjes: [3].

The classical one-dimensional continued fraction algorithm applied on a real number $x$ generates a sequence $\left(\xi_{s}\right)_{s \in \mathbb{N}}$ in $\mathbb{R}$, with $\xi_{0}=x$, named the "complete quotients", and Lagrange proved that the following assertions are equivalent:

(1) $x$ is a quadratic algebraic number.

(2) There exist natural numbers $s \geq 0$ and $p \geq 1$ such that $\xi_{s+p}=\xi_{s}$.

Manuscrit reçu le 21 avril 2012, révisé le 7 avril 2013, accepté le 14 mars 2014.

Classification math. 11J70, 11J13, $11 \mathrm{H} 06$. 
(3) There exist natural numbers $s_{0} \geq 0$ and $p \geq 1$ such that for every $s \geq s_{0}, \xi_{s+p}=\xi_{s}$ holds (periodicity).

The property $(\mathbf{2})$ will be called loop property in this paper.

Here we define a very natural two-dimensional continued-fraction algorithm for which the analogue in dimension two of the properties (1) and (2) are equivalent: This algorithm, named Smallest Vector Algorithm or " $S V A$ ", makes a loop (property (2)) if and only if the real numbers which are its two initial values are in the same cubic field (property (1)). The $S V A$ is defined at the beginning of Subsection 1.3..

We have to notice that we do not have periodicity, i.e. the property (3), for initial values in the same cubic fields. The reason why is that our algorithm, unlike a lot of known multidimensional continued fraction algorithms, is not of the vectorial kind. Therefore, the loop property (2) does not imply periodicity (3). Nevertheless, the loop property (2) implies interesting algebraic properties and the fact that the algorithm is not vectorial permits strong approximation properties.

Let's state our Lagrange-type theorem. From any initial value $\mathbf{X}_{0}=$ $\mathbf{X}={ }^{\mathrm{T}}\left(x_{0}, x_{1}, x_{2}\right)$, with $0<x_{0}<x_{1}<x_{2}$, the Smallest Vector Algorithm generates a sequence $\left(\mathbf{X}_{s}\right)=\left({ }^{\mathrm{T}}\left(x_{0, s}, x_{1, s}, x_{2, s}\right)\right)$ of triplets of real numbers, and we have the following statement.

Theorem 1 (Lagrange Loop Theorem).

First Part: Let $\rho$ be any real root of a third degree irreducible polynomial $P(r)=r^{3}-a r^{2}-b r-c$, with $a, b, c$ rationals; let $\mathbf{X}={ }^{\mathrm{T}}\left(x_{0}, x_{1}, x_{2}\right)$ be any rationally independent triplet of real numbers in the field $\mathbb{Q}[\rho]$, with $0<x_{0}<x_{1}<x_{2}$. Then the Smallest Vector Algorithm applied on the triplet $\mathbf{X}$ "makes a loop": there exist integers $s$ and $p$ with $p>0$ and a real number $\lambda$ such that:

$$
\mathbf{X}_{s+p}=\lambda \mathbf{X}_{s} \text { or equivalently: } \mathbf{x}_{s+p}=\mathbf{x}_{s} \text {, with } \mathbf{x}_{s}=\left(\frac{x_{0, s}}{x_{2, s}}, \frac{x_{1, s}}{x_{2, s}}\right) \text {. }
$$

Moreover, $\lambda$ is an algebraic integer of degree 3, and a unit, such that $\mathbb{Q}[\rho]=$ $\mathbb{Q}[\lambda]$. The minimal polynomial of $\lambda$ can be easily deduced from the relation $\mathbf{X}_{s+p}=\lambda \mathbf{X}_{s}$, as also the expressions of $\frac{x_{0}}{x_{2}}$ and $\frac{x_{1}}{x_{2}}$ as rational fractions of $\lambda$.

Second Part (Converse Statement) : Let $\mathrm{X}={ }^{\mathrm{T}}\left(x_{0}, x_{1}, x_{2}\right)$ be any rationally independent triplet of real numbers, with $0<x_{0}<x_{1}<x_{2}$. Let's suppose that the Smallest Vector Algorithm applied on the triplet $\mathbf{X}$ makes "a loop"i.e. that $\mathbf{X}_{s+p}=\lambda \mathbf{X}_{s}$ with $p>0$. Then $\lambda$ is an algebraic integer of degree 3, and a unit. Again, the minimal polynomial of $\lambda$ can be easily deduced from the relation $\mathbf{X}_{s+p}=\lambda \mathbf{X}_{s}$, as also the expressions of $\frac{x_{0}}{x_{2}}$ and $\frac{x_{1}}{x_{2}}$ as rational fractions of $\lambda$. 
The objects in this theorem are more precisely described in the following subsections. We also prove that the same algorithm provides rational approximations with Dirichlet properties, id est, with an optimal exponent.

Throughout this paper, we are going to use only the canonical euclidean norm and inner product in $\mathbb{R}^{3}$ for our approximations.

Our Dirichlet property is that for every independent triplet of positive real numbers $\mathbf{X}={ }^{\mathrm{T}}\left(x_{0}, x_{1}, x_{2}\right)$, the algorithm generates a sequence $\left(\mathbf{g}_{0}^{(s)}, \mathbf{g}_{1}^{(s)}, \mathbf{g}_{2}^{(s)}\right)$ of triplets of three-dimensional integer vectors, which realize integer approximation of the plane $\left(\mathbf{X}^{\perp}\right)$ with the following inequality, on an infinite set $S$ of integers:

$$
\sup _{s \in S}\left[\left(\min _{i=0,1,2}\left|\mathbf{g}_{i}^{(s)} \bullet \mathbf{X}\right|\right)\left(\max _{i=0,1,2}\left\|\mathbf{g}_{i}^{(s)}\right\|\right)^{2}\right]<+\infty
$$

(the index ${ }^{(s)}$ is above, in parentheses; the big point denotes the scalar product), with of course: $\lim _{s \rightarrow+\infty, s \in S}\left(\max _{i=0,1,2}\left\|\mathbf{g}_{i}^{(s)}\right\|\right)=+\infty$. See Theorem 2 in subsection 1.3.. We prove additional Dirichlet properties, for the integer approximation of $\mathbb{R} \mathbf{X}$ as well as of $\mathbf{X}^{\perp}$, when $\mathbf{X}^{\perp}$ (or $\mathbf{X}$ ) has a bad approximation property. See again subsection 1.3..

Let's notice that the approximation properties of our algorithm hold only for a subsequence of the integer vectors $\left(\mathbf{g}_{0}^{(s)}, \mathbf{g}_{1}^{(s)}, \mathbf{g}_{2}^{(s)}\right)_{s \in \mathbb{N}}$; the algorithm has a very simple geometrical definition, and strong geometrical, algebraic and approximation properties, but it is not designed to provide only best approximants, or only approximants with optimal exponent.

The goal of this paper is also to show the relations between different kinds of properties of such an algorithm:

(a) Lagrange property;

(b) Dirichlet approximation properties;

(c) Best approximation properties.

(d) Properties of the triplet $X$ which is the initial value of the algorithm (it may be badly approximable by integers, or well approximable)

(e) Geometrical properties of the tetrahedrons formed by the three integer vectors, generated at each step by the algorithm.

This study is a generalization of the well known continued fractions theory in dimension 1, with a two-dimensional algorithm which has more properties than most of the existing ones. Let's notice that all the mathematical techniques used in this paper are elementary. The most sophisticated tool appearing here is the Minkowski's Theorem on Successive Minima of symmetrical convex sets. 
At the end of the paper, in Section 6., the reader shall find a short review of the themes on Diophantine approximation which are related to this paper, with some bibliographical references.

In subsection 1.3. are given the main theorems and definitions of this paper. In subsection 1.4. the reader shall find two numerical examples of Lagrange loops. The plan of our paper is in subsection 1.5.

But first, in order to understand better the two-dimensional case, we recall some facts and notations about the classical continued fractions algorithm in dimension one, from a particular point of view.

\subsection{The one dimensional example.}

1.2.1. A formalism with matrices. A real number $x$ is chosen, with $0<x<1$. Here it is supposed to be irrational. Let the vector $\mathbf{X}$ be: $\mathbf{X}={ }^{\mathrm{T}}(x, 1)$, where the "T" denotes the transposition. The classic onedimensional algorithm provides integer points ${ }^{\mathrm{T}}\left(p_{n}, q_{n}\right)$ which are the nearest integer points to the line $\mathbb{D}=\mathbb{R} \mathbf{X}$. These points are called "convergent" points. We consider the matrices $\mathbf{B}_{n}=\left(\begin{array}{ll}p_{n-1} & p_{n} \\ q_{n-1} & q_{n}\end{array}\right)$. We have the relation: $\mathbf{B}_{n+1}=\mathbf{B}_{n} \times\left(\begin{array}{cc}0 & 1 \\ 1 & a_{n}\end{array}\right)$, where $a_{n}$ is the $n$-th "partial quotient" of the continued fraction and is a strictly positive integer. If we denote: $\mathbf{A}_{n}=\left(\begin{array}{cc}0 & 1 \\ 1 & a_{n}\end{array}\right)$, then the approximating matrices $\mathbf{B}_{n}$ appear as products of matrices $\mathbf{A}_{k}(1 \leq k \leq n-1)$. Let's notice that $\mathbf{B}_{1}$ is the Identity matrix.

In order to be closer to our two-dimensional algorithm, we may also split the $n$-th step into more elementary steps, and consider the simple matrix $\mathbf{D}=\left(\begin{array}{ll}1 & 0 \\ 1 & 1\end{array}\right)$, then we have $\mathbf{B}_{n+1}=\mathbf{B}_{n} \mathbf{A}_{n}=\mathbf{B}_{n} \mathbf{D}^{a_{n}}\left(\begin{array}{ll}0 & 1 \\ 1 & 0\end{array}\right)$. The last matrix corresponds to an exchange of vectors, when the following convergent ${ }^{\mathrm{T}}\left(p_{n+1}, q_{n+1}\right)$ is found. We may notice that all the matrices involved have determinant \pm 1 .

1.2.2. Polar matrices, cofactors, periodicity. We also introduce the polar matrices $\mathbf{G}_{n}$, each of them being the transposed matrix of the inverse of $\mathbf{B}_{n}$. Let $\mathbf{g}_{0, n}$ and $\mathbf{g}_{1, n}$ be the column vectors of $\mathbf{G}_{n}$. These vectors realize integer approximations of the line $\boldsymbol{\Delta}$ orthogonal to $\mathbb{D}$, and the regular continued fraction algorithm is precisely designed to obtain both: $\mathbf{g}_{0, n} \bullet \mathbf{X}>$ 0 and $\mathbf{g}_{1, n} \bullet \mathbf{X}>0$ for the scalar products.

These quantities $\mathbf{g}_{0, n} \bullet \mathbf{X}$ and $\mathbf{g}_{1, n} \bullet \mathbf{X}$ are particularly important in the theory of continued fractions. Let $\mathbf{b}_{0, n}$ and $\mathbf{b}_{1, n}$ be the column vectors of $\mathbf{B}_{n}$. We have the vectorial relation: $\left(\mathbf{g}_{0, n} \bullet \mathbf{X}\right) \mathbf{b}_{0, n}+\left(\mathbf{g}_{1, n} \bullet \mathbf{X}\right) \mathbf{b}_{1, n}=\mathbf{X}$. (To see that, make the scalar product of the left-hand vector of the equality with 
$\mathbf{g}_{0, n}$, and then with $\left.\mathbf{g}_{1, n}\right)$. Because of this relation, $\left(\mathbf{g}_{0, n} \bullet \mathbf{X}\right)$ and $\left(\mathbf{g}_{1, n} \bullet \mathbf{X}\right)$ are called the cofactors in the algorithm.

Let's form the cofactors vector: $\mathbf{X}_{n}=\left(\begin{array}{l}\mathbf{g}_{0, n} \bullet \mathbf{X} \\ \mathbf{g}_{1, n} \bullet \mathbf{X}\end{array}\right)$. We have:

$$
{ }^{\mathrm{T}} \mathbf{G}_{n} \mathbf{X}=\mathbf{X}_{n}, \text { id est } \mathbf{B}_{n}^{-1} \mathbf{X}=\mathbf{X}_{n}
$$

Now we may calculate $\mathbf{X}_{n}$. We have $\mathbf{B}_{n}^{-1}=(-1)^{(n-1)}\left(\begin{array}{cc}q_{n} & -p_{n} \\ -q_{n-1} & p_{n-1}\end{array}\right)$ and then

$$
\mathbf{X}_{n}=\mathrm{T}\left((-1)^{n}\left(p_{n}-x q_{n}\right),(-1)^{(n-1)}\left(p_{n-1}-x q_{n-1}\right)\right) .
$$

Then we define the sequence $\left(x_{n}\right)$ by $x_{n}=(-1)^{n}\left(p_{n}-x q_{n}\right)=\mathbf{g}_{0, n} \bullet \mathbf{X}$. At the first step, $x_{1}=x$.

We may now give the rule which defines the quantity $a_{n}$, in dimension 1 . Here the brackets denote the integer part: $a_{n}=\left[\frac{\mathbf{g}_{1, n} \bullet \mathbf{X}}{\mathbf{g}_{0, n} \bullet \mathbf{X}}\right]=\left[\frac{x_{n-1}}{x_{n}}\right]$.

We now define another object, the inverse of this quotient: $\xi_{n}:=\frac{x_{n}}{x_{n-1}}$. Then the preceding relation writes: $a_{n}=\left[\frac{1}{\xi_{n}}\right]$.

The quantities $x_{n}$ and the cofactors vectors $\mathbf{X}_{n}$ are of highest interest in questions concerning Lagrange property. This algorithm is eventually periodic, from the range $n$, if and only if there exist an integer $p \geq 1$ and a real number $\lambda$ such that $\mathbf{X}_{n+p}=\lambda \mathbf{X}_{n}$, or equivalently: $\xi_{n+p}=\xi_{n}$. These are the conditions we shall use in our Lagrange Theorem.

1.2.3. Recursive relations on the polar matrices. Let's denote by $\mathbf{M}^{*}$ the polar matrix of $\mathbf{M}$, such that $\mathbf{M}^{*}=\left({ }^{\mathrm{T}} \mathbf{M}\right)^{-1}$ We have $\mathbf{G}_{n}=\mathbf{B}_{n}^{*}$, and then, each matrix $\mathbf{B}_{n}$ is a product of matrices $\mathbf{A}_{k}^{*}(1 \leq k \leq n-1)$, with $\mathbf{A}_{k}^{*}=\left(\begin{array}{cc}-a_{k} & 1 \\ 1 & 0\end{array}\right)$. We may consider the simpler matrix $\mathbf{C}=\left(\begin{array}{cc}1 & 0 \\ -1 & 1\end{array}\right)$, then we have $\mathbf{G}_{n+1}=\mathbf{G}_{n} \mathbf{A}_{n}^{*}=\mathbf{G}_{n} \mathbf{C}^{a_{n}}\left(\begin{array}{ll}0 & 1 \\ 1 & 0\end{array}\right)$.

This leads to the relations ${ }^{\mathrm{T}} \mathbf{G}_{n+1}=\mathbf{A}_{n}^{-1}{ }^{\mathrm{T}} \mathbf{G}_{n}$ and then, by ${ }^{\mathrm{T}} \mathbf{G}_{n} \mathbf{X}=$ $\mathbf{X}_{n}$, to: $\mathbf{X}_{n+1}=\mathbf{A}_{n}^{-1} \mathbf{X}_{n}=\left(\begin{array}{cc}-a_{n} & 1 \\ 1 & 0\end{array}\right) \mathbf{X}_{n}$; therefore: $x_{n+1}=x_{n-1}-a_{n} x_{n}$. Because of this formula, the continued fractions algorithms may be called subtractive. This leads to the recursive relation: $\xi_{n+1}=\frac{1}{\xi_{n}}-a_{n}=\frac{1}{\xi_{n}}-\left[\frac{1}{\xi_{n}}\right]$.

In particular, if $\mathbf{g}_{0}$ and $\mathbf{g}_{1}$ are the column vectors of $\mathbf{G}$, then the column vectors of the following matrix $\mathbf{G} \times \mathbf{C}=\mathbf{G} \times\left(\begin{array}{cc}1 & 0 \\ -1 & 1\end{array}\right)$ are $\left(\mathbf{g}_{0}-\mathbf{g}_{1}\right)$ and $\mathbf{g}_{1}$. Our two-dimensional algorithm is built in a similar way, in the next subsection. 
1.2.4. Use of the orthogonal projections. From a more geometrical point of view, let's denote by $\mathbf{g}_{0, n}^{\prime \prime}$ and $\mathbf{g}_{1, n}^{\prime \prime}$ the orthogonal projections of $\mathbf{g}_{0, n}$ and $\mathbf{g}_{1, n}$ on $\mathbb{D}$. Then we also have: $\mathbf{X}_{n}=\|\mathbf{X}\|\left(\left\|\begin{array}{l}\mathbf{g}_{0, n}^{\prime \prime} \\ \mathbf{g}_{1, n}^{\prime \prime}\end{array}\right\|\right)$.

Concerning a Dirichlet property of the algorithm, it can be written:

For each $n,\left\|\mathbf{g}_{1, n}^{\prime \prime}\right\| q_{n} \leq 1$. Our matricial formalism would permit to prove easily the Lagrange Theorem for a quadratic number $x$, using this Dirichlet property.

Now we are going to generalize all these properties and demonstrations in dimension two.

1.3. Results in this paper. Now we are in dimension two. Throughout this paper we suppose that the triplet $\mathbf{X}={ }^{\mathrm{T}}\left(x_{0}, x_{1}, x_{2}\right)$ of real numbers is rationally independent, and verifies $0<x_{0}<x_{1}<x_{2}$. We use the canonical euclidean norm in $\mathbb{R}^{3}$ and the canonical scalar product. We denote by $\mathbb{D}$ the line $\mathbb{D}=\mathbb{R} \mathbf{x}$ and by $\mathbb{P}$ the plane orthogonal to $\mathbb{D}$.

As it is usually done in this field, the index ${ }^{(s)}$ of the sequences will be above, in parentheses.

Definition. The Smallest Vector Algorithm is described by the following sequence $\left(\mathbf{G}^{(s)}\right)_{s \in \mathbb{N}}$ of $3 \times 3$ integer matrices, which is inductively defined by:

a) $\mathbf{G}^{(0)}=\mathbf{I}$, the Identity matrix.

b) Let's suppose $\mathbf{G}^{(s)}=\left(\mathbf{g}_{0}^{(s)}, \mathbf{g}_{1}^{(s)}, \mathbf{g}_{2}^{(s)}\right)$ has been defined. Let $\mathbf{g}_{i}^{(s)}$ and $\mathrm{g}^{\prime \prime}{ }_{i}^{(s)}$ denote the respective orthogonal projections of $\mathbf{g}_{i}^{(s)}$ on $\mathbb{D}$ and $\mathbb{P}$. Let $\Delta_{\text {min }}$ denote $\Delta_{\min }:=\min \left(\left\|\mathbf{g}_{1}^{(s)}-\mathbf{g}_{0}^{\prime(s)}\right\|,\left\|\mathbf{g}_{2}^{\prime(s)}-\mathbf{g}_{1}^{\prime(s)}\right\|,\left\|\mathbf{g}_{2}^{\prime(s)}-\mathbf{g}_{0}^{\prime(s)}\right\|\right)$. We define first the three column vectors $\left(\mathbf{f}_{0}, \mathbf{f}_{1}, \mathbf{f}_{2}\right)$ of $\mathbf{G}^{(s+1)}$, in disorder:

$$
\begin{gathered}
\left(\mathbf{f}_{0}, \mathbf{f}_{1}, \mathbf{f}_{2}\right)=\left(\mathbf{g}_{0}^{(s)}, \mathbf{g}_{1}^{(s)}-\mathbf{g}_{0}^{(s)}, \mathbf{g}_{2}^{(s)}\right) \text { if } \Delta_{\min }=\left\|\mathbf{g}_{1}^{\prime(s)}-\mathbf{g}_{0}^{\prime(s)}\right\| ; \\
\left(\mathbf{f}_{0}, \mathbf{f}_{1}, \mathbf{f}_{2}\right)=\left(\mathbf{g}_{0}^{(s)}, \mathbf{g}_{1}^{(s)}, \mathbf{g}_{2}^{(s)}-\mathbf{g}_{1}^{(s)}\right) \text { if } \Delta_{\min }=\left\|\mathbf{g}_{2}^{\prime(s)}-\mathbf{g}_{1}^{\prime(s)}\right\| ; \\
\left(\mathbf{f}_{0}, \mathbf{f}_{1}, \mathbf{f}_{2}\right)=\left(\mathbf{g}_{0}^{(s)}, \mathbf{g}_{1}^{(s)}, \mathbf{g}_{2}^{(s)}-\mathbf{g}_{0}^{(s)}\right) \text { if } \Delta_{\min }=\left\|\mathbf{g}_{2}^{(s)}-\mathbf{g}_{0}^{\prime(s)}\right\| . \\
\mathbf{G}^{(s+1)}=\left(\mathbf{g}_{0}^{(s+1)}, \mathbf{g}_{1}^{(s+1)}, \mathbf{g}_{2}^{(s+1)}\right) \text { is defined as any of the permutations of }
\end{gathered}
$$
the vectors $\left(\mathbf{f}_{0}, \mathbf{f}_{1}, \mathbf{f}_{2}\right)$ such that we have: $\left\|\mathbf{g}_{0}^{\prime \prime(s+1)}\right\| \leq\left\|\mathbf{g}^{\prime \prime}{ }_{1}^{(s+1)}\right\| \leq\left\|\mathbf{g}_{2}^{\prime \prime}{ }^{(s+1)}\right\|$.

The columns $\mathbf{g}_{0}^{(s)}, \mathbf{g}_{1}^{(s)}, \mathbf{g}_{2}^{(s)}$ of the matrices $\mathbf{G}^{(s)}$ realize integer approximations of the plane $\mathbb{P}$. They play the same role in dimension 2 as, in dimension 1, the matrices $\mathbf{G}_{n}$ studied above. 
As in dimension 1 , the cofactors vector $\mathbf{X}^{(s)}$ is fundamental. It's defined by: $\mathbf{X}^{(s)}=\|\mathbf{X}\| \cdot{ }^{\mathrm{T}}\left(\left\|\mathbf{g}_{0}^{\prime \prime(s)}\right\|,\left\|\mathbf{g}_{1}^{\prime \prime(s)}\right\|,\left\|\mathbf{g}_{2}^{\prime \prime(s)}\right\|\right)$, and the vector $\mathbf{x}^{(s)}$, the "projective" version of $\mathbf{X}^{(s)}$, is defined by $\mathbf{x}^{(s)}=\mathrm{T}\left(\frac{\left\|\mathbf{g}_{0}^{\prime \prime(s)}\right\|}{\left\|\mathbf{g}_{2}^{\prime \prime(s)}\right\|}, \frac{\left\|\mathbf{g}_{1}^{\prime \prime(s)}\right\|}{\left\|\mathbf{g}_{2}^{\prime \prime(s)}\right\|}, 1\right)$.

The Smallest Vector Algorithm has a Lagrange property, which is expressed by Theorem 1 of subsection 1.1. The demonstration of this theorem (see below) is essentially the same as in dimension one, based on a Dirichlet result (In dimension two, Theorem 2).

First we introduce the unimodular positive integer matrix $\mathbf{B}^{(s)}$, defined as the polar matrix of $\mathbf{G}^{(s)}$ (the transposed matrix of the inverse of $\mathbf{G}^{(s)}$ ). We denote by $\mathbf{b}_{0}^{(s)}, \mathbf{b}_{1}^{(s)}, \mathbf{b}_{2}^{(s)}$ the column vectors of $\mathbf{B}^{(s)}$ which realize integer approximations of the line $\mathbb{D}$. The vectors $\mathbf{b}_{0}^{\prime \prime(s)}, \mathbf{b}_{1}^{\prime \prime(s)}, \mathbf{b}_{1}^{\prime \prime(s)}$ will be the orthogonal projections of these vectors on $\mathbb{D}$, and the $\mathbf{b}_{i}^{\prime(s)}$ their orthogonal projections on $\mathbb{P}$. In the same way are defined the $\mathbf{g}_{1}^{\prime \prime(s)}$ and the $\mathbf{g}_{i}^{\prime(s)}$, and, for any vector $\mathbf{h}$, the projections $\mathbf{h}^{\prime}$ and $\mathbf{h}^{\prime \prime}$ of $\mathbf{h}$ on $\mathbb{P}$ and $\mathbb{D}$,

Theorem 2 (Dirichlet Properties). For each rationally independent triplet $\mathbf{X}$ of real numbers, with $0<x_{0}<x_{1}<x_{2}$, the Smallest Vector Algorithm has the following properties

a) There exists an infinite set $S$ of natural integers such that:

$$
\begin{gathered}
\sup _{s \in S}\left[\left(\max _{i=0,1,2}\left\|\mathbf{g}_{i}^{\prime(s)}\right\|\right)^{2}\left(\min _{i=0,1,2}\left\|\mathbf{g}_{i}^{\prime \prime(s)}\right\|\right)\right]<+\infty, \text { with also: } \\
\lim _{s \rightarrow+\infty, s \in S}\left(\min _{i=0,1,2}\left\|\mathbf{g}_{i}^{\prime \prime(s)}\right\|\right)=0 .
\end{gathered}
$$

In other words, this algorithm provides a Diophantine approximation of $\mathbb{P}$ which possesses a Dirichlet property, with the optimal exponent, 2 , and with a form which is rather strong.

b) If there exists $c>0$ such that for any integer point $\mathbf{h} \neq \mathbf{0}$, $\|\mathbf{h}\|^{2}\left\|\mathbf{h}^{\prime \prime}\right\|>$ c holds (id est, if the couple $\left(\frac{x_{1}}{x_{0}}, \frac{x_{2}}{x_{0}}\right)$ is badly approximable, as it is proved in Section 3), then the following relation holds with the Smallest Vector Algorithm, with the same infinite set $S$ :

$$
\begin{gathered}
\sup _{s \in S}\left[\left(\max _{i=0,1,2}\left\|\mathbf{g}_{i}^{\prime(s)}\right\|\right)^{2}\left(\max _{i=0,1,2}\left\|\mathbf{g}_{i}^{\prime \prime(s)}\right\|\right)\right]<+\infty, \text { with also: } \\
\lim _{s \rightarrow+\infty, s \in S}\left(\max _{i=0,1,2}\left\|\mathbf{g}_{i}^{\prime \prime(s)}\right\|\right)=0 .
\end{gathered}
$$


c) If again there exists $c>0$ such that for any integer point $\mathbf{h} \neq \mathbf{0}$, $\|\mathbf{h}\|^{2}\left\|\mathbf{h}^{\prime \prime}\right\|>c$ holds, then

$$
\begin{gathered}
\sup _{s \in S}\left[\left(\max _{i=0,1,2}\left\|\mathbf{b}_{i}^{\prime(s)}\right\|\right)^{2}\left(\max _{i=0,1,2}\left\|\mathbf{b}_{i}^{\prime \prime(s)}\right\|\right)\right]<+\infty ; \text { with also: } \\
\lim _{s \rightarrow+\infty, s \in S}\left(\max _{i=0,1,2}\left\|\mathbf{b}_{i}^{\prime(s)}\right\|\right)=0 .
\end{gathered}
$$

Theorem 3 (Geometrical Theorem). The Smallest Vector Algorithm possesses the following property. Let $\mathbf{H}^{\prime(s)}$ be the convex hull

$$
\mathbf{H}^{\prime(s)}=\operatorname{CONV}\left(\left\{\mathbf{g}_{0}^{\prime(s)}, \mathbf{g}_{1}^{\prime(s)}, \mathbf{g}_{2}^{\prime(s)},-\mathbf{g}_{0}^{\prime(s)},-\mathbf{g}_{1}^{\prime(s)}-, \mathbf{g}_{2}^{\prime(s)}\right\}\right) \text { in } \mathbb{P} .
$$

Let $\rho^{(s)}$ be the radius of the greatest disk in $\mathbb{P}$ with center 0 contained in $\mathbf{H}^{\prime(s)}$. Then there exists an infinite set $S$ of natural integers such that:

$$
\sup _{s \in S} \frac{\max _{i=0,1,2}\left\|\mathbf{g}_{i}^{\prime(s)}\right\|}{\rho^{(s)}}<+\infty
$$

Definition. If $\liminf _{s \rightarrow+\infty} \frac{\max _{i=0,1,2}\left\|\mathbf{g}_{i}^{(s)}\right\|}{\rho^{(s)}}<+\infty$, with the notations of the above Geometrical Theorem, it will be said that the algorithm is balanced.

As a Best Approximation result, we give our Prism Lemma, which is proved in Subsection 3.1.. It is a very easy result, but it is true and important for any continued fraction algorithm, and the author has not seen this statement anywhere in literature.

Lemma (Prism Lemma). Let $\mathbf{g}_{0}^{(s)}, \mathbf{g}_{1}^{(s)}, \mathbf{g}_{2}^{(s)}$ be the column vectors of the matrix $\mathbf{G}^{(s)}$ generated at the s-th step by the Smallest Vector Algorithm. Let the sets $\mathbf{H}^{\prime(s)}$ be the convex hulls:

$$
\mathbf{H}^{\prime(s)}=\operatorname{CONV}\left(\mathbf{g}_{0}^{\prime(s)}, \mathbf{g}_{1}^{\prime(s)}, \mathbf{g}_{2}^{\prime(s)},-\mathbf{g}_{0}^{\prime(s)},-\mathbf{g}_{1}^{\prime(s)},-\mathbf{g}_{2}^{\prime(s)}\right) \text { in } \mathbb{P} .
$$

We shall omit the indices ${ }^{(s)}$. Let $\mathbf{H}$ be the prism: $\mathbf{H}=\mathbf{H}^{\prime}+\mathbb{D}$. Then, with the usual notation $\mathbf{h}^{\prime \prime}$ for the orthogonal projection of $\mathbf{h}$ on $\mathbb{D}$ :

-For each non zero integer point $h$ in $\mathbf{H},\left\|\mathbf{h}^{\prime \prime}\right\| \geq\left\|\mathbf{g}_{0}^{\prime \prime}\right\|$ holds.

-For each integer point $\mathbf{h}$ in $\mathbf{H}$ which is not of the form $n_{0} \mathbf{g}_{0}$, with $n_{0}$ integer, $\left\|\mathbf{h}^{\prime \prime}\right\| \geq\left\|\mathbf{g}_{1}^{\prime \prime}\right\|$ holds.

-For each integer point $\mathbf{h}$ in $\mathbf{H} \quad$ which is not of the form $n_{0} \mathbf{g}_{0}+n_{1} \mathbf{g}_{1}$, with $n_{0}$ and $n_{1}$ integers, $\left\|\mathbf{h}^{\prime \prime}\right\| \geq\left\|\mathbf{g}_{2}^{\prime \prime}\right\|$ holds.

- That implies that, if $\left(\mathbf{h}_{0}, \mathbf{h}_{1}, \mathbf{h}_{2}\right)$ is a free triplet of integer points in $\mathbf{H}$, then $\max _{i=0,1,2}\left(\left\|\mathbf{h}_{i}^{\prime \prime}\right\|\right) \geq \max _{i=0,1,2}\left(\left\|\mathbf{g}_{i}^{\prime \prime}\right\|\right)=\left\|\mathbf{g}_{2}^{\prime \prime}\right\|$. 
The previous theorems suppose that the initial values $\left(x_{0} ; x_{1} ; x_{2}\right)$ of our algorithm are rationally independent. The reader may wonder what happens when they are not.

Theorem 4. The Smallest Vector Algorithm finds rational dependence. If the initial values $\left(x_{0}, x_{1}, x_{2}\right)$ of our algorithm are rationally dependent, then the SVA generates at some step an integer triplet $\left(\mathbf{g}_{0}^{(s)}, \mathbf{g}_{1}^{(s)}, \mathbf{g}_{2}^{(s)}\right)$ such that: $\left\|\mathbf{g}_{0}^{\prime \prime(s)}\right\|=\mathbf{g}_{0}^{(s)} \bullet \mathbf{X}=0$. This relation gives the coefficients of the rational (integral) dependence of $\left(x_{0}, x_{1}, x_{2}\right)$.

The proof uses Lemma 2 in the next subsection, the Geometrical Theorem and the Prism Lemma. The demonstrations of these three results are still valid if $\left(x_{0}, x_{1}, x_{2}\right)$ is rationally dependent. With these three results, the proof of Theorem 4 is very short.

Proof. Let's suppose that we have an integral dependence relation, of the shape $\mathbf{h} \bullet \mathbf{X}=0, \mathbf{h}$ being a non null integer vector. By the Lemma 2 of the next subsection: $\lim _{s \rightarrow+\infty}\left(\max _{i=0,1,2}\left\|\mathbf{g}_{i}^{\prime(s)}\right\|\right)=+\infty$. In addition, by the Geometrical Theorem above, $\liminf _{s \rightarrow+\infty} \frac{\max _{i=0,1,2}\left\|\mathbf{g}_{i}^{(s)}\right\|}{\rho^{(s)}}<+\infty$. Then $\limsup _{s \rightarrow+\infty} \rho^{(s)}=+\infty$. Then $\mathbf{h}$ belongs to some hexagon $\mathbf{H}^{\prime(s)}$ defined as a convex hull in the Prism Lemma just above. By this Prism Lemma, $0=\left\|\mathbf{h}^{\prime \prime}\right\| \geq\left\|\mathbf{g}_{0}^{\prime \prime}\right\|=\frac{\mathbf{X} \bullet \mathbf{g}_{0}^{(s)}}{\|\mathbf{X}\|} \geq 0$ holds. Then $\mathbf{X} \bullet \mathbf{g}_{0}^{(s)}=0$.

1.4. Numerical examples. We give two examples of Lagrange Loops when the initial values are in some cubic field.

Example. $\left(x_{0} ; x_{1} ; x_{2}\right)=\left(1 ; 2 \cos (\pi / 7) ; 4 \cos ^{2}(\pi / 7)\right)$. The sequence of the $\left(\frac{x_{0}^{(s)}}{x_{2}^{(s)}} ; \frac{x_{1}^{(s)}}{x_{2}^{(s)}}\right)$ is the simplest the author has met. Almost every couple in it repeats infinitely. We have: $x_{1}^{3}-x_{1}^{2}-2 x_{1}+1=0$, and $\left(x_{2}-2\right)^{3}+\left(x_{2}-2\right)^{2}-$ $2\left(x_{2}-2\right)-1=0$; the algorithm provides the following $\left(s+1,\left(\frac{x_{0}^{(s)}}{x_{2}^{(s)}} ; \frac{x_{1}^{(s)}}{x_{2}^{(s)}}\right)\right)$.

\begin{tabular}{|c|c|}
\hline & \\
\hline 80193773580484, & $3.24697960371747\}\}$ \\
\hline$\{2,\{1.80193773580484$, & $2.24697960371747\}$ \\
\hline$\{3,\{1.24697960371747$, & $2.80193773580484\}$ \\
\hline$\{4,\{1.24697960371747$ & $1.80193773580484\}$ \\
\hline $\begin{array}{l}\{5,\{1.80193773580484, \\
\ldots / \ldots\end{array}$ & $2.24697960371747\}$ \\
\hline$\left\{292,\left\{1.2469796037174^{\prime}\right.\right.$ & 1.80193773580484 \\
\hline$\{293,\{1.80193773580484$ & 2.246979603717 \\
\hline
\end{tabular}


$\{294,\{1.24697960371747$, $1.80193773580484\}\}$ $\{295,\{1.24697960371747$, $1.55495813208737\}\}$ $\{296,\{1.80193773580484$, $2.24697960371747\}\}$ $\{297,\{1.24697960371747$, $1.80193773580484\}\}$ $\{298,\{1.80193773580484$, $2.24697960371747\}\}$ $\{299,\{1.24697960371747$, $2.80193773580484\}\}$ $\{300,\{1.24697960371747$, $1.80193773580484\}\}$

Example. $\left(x_{0} ; x_{1} ; x_{2}\right)=\left(1 ; \sqrt[3]{13} ; \sqrt[3]{13^{2}}\right)$. The algorithm provides the following sequence $\left(s+1,\left(\frac{x_{0}^{(s)}}{x_{2}^{(s)}} ; \frac{x_{1}^{(s)}}{x_{2}^{(s)}}\right)\right)$. Here there are very few repetitions (loops), but they exist.
$\{1,\{2.35133468772075748950001$,
$5.5287748136788721414723\}\}$
$\{2,\{2.35133468772075748950001$,
$4.5287748136788721414723\}\}$
$\{3,\{1.35133468772075748950001$,
$4.5287748136788721414723\}\}$ .../...
$\{104,\{2.35133468772075748950001$,
$5.528774813678872141472\}\}$
$\{105,\{2.35133468772075748950001$,
$4.528774813678872141472\}\}$
$\{106,\{1.35133468772075748950001$, ... $/ .$.
$\{160,\{5.5057084068852398563646$, .../...
$4.528774813678872141472\}\}$
$\{219,\{1.35133468772075748950001$, .../...
$\{308,\{2.35133468772075748950001$, .../...
$\{411,\{2.35133468772075748950001$,
$47.82563987973201144317\}\}$
$4.5287748136788721414723\}\}$
$5.5287748136788721414723\}\}$
$5.5287748136788721414723\}\}$

1.5. PLAN of the paper. We shall prove the Results above in the order in which they are written in this first section, and, in fact, in the reverse of the logical order.

- In the second section, we admit that the Smallest Vector Algorithm or $S V A$ has the Dirichlet Properties of Theorem 2, and we prove that this implies the Lagrange properties. But this demonstration is made only in a particular case, namely $\mathbf{X}=$ $\left(1, \sqrt[3]{N}, \sqrt[3]{N^{2}}\right)$ with $N$ natural. The complete proof of the Lagrange Property for three numbers in a cubic number field is given in Section 5. But this general demonstration is intricate, and the particular case gives all the main ideas involved. That's why we prefer to begin with this particular case, for more clarity.

- In the third section, we admit the geometrical property of the SVA, namely that it is balanced. We prove that this property implies the Dirichlet Properties. 
- In the fourth section, we prove the Geometrical Theorem, namely that the SVA is balanced.

- In the fifth section, we give the general demonstration of the Lagrange Theorem. Then all theorems are proved.

- In Section 6, we locate the results of this paper in relation to the main themes in Multidimensional Dimensional Fractions Theory and Homogeneous Diophantine Approximation, with some bibliographical references.

\section{Demonstration of Lagrange Properties (particular case)}

2.1. Four basic Lemmas. We're going to need the following lemmas.

Lemma 1 (Basic Properties of Brentjes' Algorithms). a) Inductively, by the way of building the Smallest Vector Algorithm, we have the following properties:

$$
\begin{aligned}
0 \leq \mathbf{X} \bullet \mathbf{g}_{0}^{(s)}=\|\mathbf{X}\|\left\|\mathbf{g}_{0}^{\prime \prime(s)}\right\| \leq \mathbf{X} \bullet \mathbf{g}_{1}^{(s)}= \\
\qquad \mathbf{X}\|\| \mathbf{g}_{1}^{\prime \prime(s)}\left\|\leq \mathbf{X} \bullet \mathbf{g}_{2}^{(s)}=\right\| \mathbf{X}\|\| \mathbf{g}_{2}^{\prime \prime(s)} \| .
\end{aligned}
$$

b) The following equality holds for every $s \in \mathbb{N}$ :

$$
\|\mathbf{X}\|\left\|\mathbf{g}_{0}^{\prime \prime(s)}\right\| \cdot \mathbf{b}_{0}^{(s)}+\|\mathbf{X}\|\left\|\mathbf{g}_{1}^{\prime \prime(s)}\right\| \cdot \mathbf{b}_{1}^{(s)}+\|\mathbf{X}\|\left\|\mathbf{g}_{2}^{\prime \prime(s)}\right\| \cdot \mathbf{b}_{2}^{(s)}=\mathbf{X}
$$

That's the reason why $\|\mathbf{X}\|\left\|\mathbf{g}_{0}^{\prime \prime(s)}\right\|,\|\mathbf{X}\|\left\|\mathbf{g}_{1}^{\prime \prime(s)}\right\|,\|\mathbf{X}\|\left\|\mathbf{g}_{1}^{\prime \prime(s)}\right\|$ are called cofactors.

c) For every $s \in \mathbb{N}:{ }^{\mathrm{T}} \mathbf{G}^{(s)} \mathbf{X}=\mathbf{X}^{(s)}$ or, which is the same:

$$
\left(\mathbf{B}^{(s)}\right)^{-1} \mathbf{X}=\mathbf{X}^{(s)}
$$

Proof. First of all, the a) Property is true at the step $s=0$. Let's suppose it's true at the step $s$. The construction of the new $\mathbf{g}_{j}^{(s+1)}$ by subtraction is always done in accordance with the order of the $\mathbf{X} \bullet \mathbf{g}_{i}^{(s)}=\|\mathbf{X}\|\left\|\mathbf{g}_{i}^{\prime \prime(s)}\right\|$. Then, at the next step, each of the $\mathbf{X} \bullet \mathbf{g}_{i}^{(s+1)}$ is positive, and then equals $\|\mathbf{X}\|\left\|\mathbf{g}_{i}^{\prime \prime(s+1)}\right\|$. The rule of the algorithm is to order these numbers at step $(s+1)$. Then the property is obtained at step $(s+1)$, and a) is true by induction. For the b) property, the equality

$$
\left(\mathbf{X} \bullet \mathbf{g}_{0}^{(s)}\right) \cdot \mathbf{b}_{0}^{(s)}+\left(\mathbf{X} \bullet \mathbf{g}_{1}^{(s)}\right) \cdot \mathbf{b}_{1}^{(s)}+\left(\mathbf{X} \bullet \mathbf{g}_{2}^{(s)}\right) \cdot \mathbf{b}_{2}^{(s)}=\mathbf{X}
$$

holds. To see that, make the scalar product of both the left-hand and the right-hand vector of the equality with each of the $\mathbf{g}_{i}^{(s)}$. This equality is the same as the equality in b). 
Because $\mathbf{X}^{(s)}=\mathrm{T}\left(\mathbf{X} \bullet \mathbf{g}_{0}^{(s)}, \mathbf{X} \bullet \mathbf{g}_{1}^{(s)}, \mathbf{X} \bullet \mathbf{g}_{2}^{(s)}\right)$, the equalities of $\left.\mathrm{c}\right)$ are obvious.

Lemma 2. For the Smallest Vector Algorithm, and more generally in any Brentjes' algorithm, we have: $\lim _{s \rightarrow+\infty}\left(\max _{i=0,1,2}\left\|\mathbf{g}_{i}^{\prime(s)}\right\|\right)=+\infty$.

Proof. If this limit does not hold, there exist a real number $M$ and an infinite set $T$ such that for any $s \in T$, and for $i=0,1,2,\left\|\mathbf{g}_{i}^{\prime(s)}\right\| \leq M$ holds. In addition, by the subtractive nature of these algorithms, the $\left\|\mathbf{g}_{i}^{\prime \prime(s)}\right\|$, $i=0,1,2$ are also bounded. Then the set of the triplets $\left(\mathbf{g}_{0}^{(s)}, \mathbf{g}_{1}^{(s)}, \mathbf{g}_{2}^{(s)}\right)$ with $s \in T$ is bounded. Then it is finite. Then there exist two distinct natural numbers $s$ and $t$ such that $\left(\mathbf{g}_{0}^{(s)}, \mathbf{g}_{1}^{(s)}, \mathbf{g}_{2}^{(s)}\right)=\left(\mathbf{g}_{0}^{(t)}, \mathbf{g}_{1}^{(t)}, \mathbf{g}_{2}^{(t)}\right)$ and particularly: $\left(\mathbf{g}_{0}^{\prime \prime(s)}, \mathbf{g}_{1}^{\prime \prime(s)}, \mathbf{g}_{2}^{\prime \prime(s)}\right)=\left(\mathbf{g}_{0}^{\prime \prime(t)}, \mathbf{g}_{1}^{\prime \prime(t)}, \mathbf{g}_{2}^{\prime \prime(t)}\right)$. But that's impossible, again by the subtractive nature of these algorithms. Our hypothesis was false, and then the conclusion of the theorem holds.

Now, we state two lemmas which will provide the Second Part of our Lagrange Theorem.

Lemma 3 (Degree in a Loop). Let $\mathbf{X}={ }^{\mathrm{T}}\left(x_{0}, x_{1}, x_{2}\right)$ be any rationally independent triplet of real numbers, with $0<x_{0}<x_{1}<x_{2}$. Let's suppose that the Smallest Vector Algorithm applied on the triplet $\mathbf{X}$ "makes a loop" i.e. that $\mathbf{X}^{(s+p)}=\lambda \mathbf{X}^{(s)}$ with $p>0$. Then $\lambda$ cannot be a quadratic real number.

Proof. If the case of such a loop, we have $\mathbf{X}^{(s+p)}=\lambda \mathbf{X}_{s}^{(s)}$, or equivalently

$$
\left(\mathbf{B}^{(s+p)}\right)^{-1} \mathbf{X}=\lambda\left(\mathbf{B}^{(s)}\right)^{-1} \mathbf{X} \text { or }\left(\mathbf{B}^{(s+p)}\right)^{-1} \mathbf{B}^{(s)} \mathbf{X}^{(s)}=\lambda \mathbf{X}^{(s)}
$$

Let's denote: $\widetilde{\mathbf{B}}:=\left(\mathbf{B}^{(s+p)}\right)^{-1} \mathbf{B}^{(s)}$. Then: $\widetilde{\mathbf{B}} \mathbf{X}^{(s)}=\lambda \mathbf{X}^{(s)}$, with

$$
\mathbf{X}^{(s)}=\mathrm{T}\left(x_{0}^{(s)}, x_{1}^{(s)}, x_{2}^{(s)}\right) .
$$

Let's denote: $\mathbf{Y}:=\mathrm{T}\left(\frac{x_{0}^{(s)}}{x_{2}^{(s)}}, \frac{x_{1}^{(s)}}{x_{2}^{(s)}}, 1\right):=\mathrm{T}\left(y_{0}, y_{1}, 1\right)$.

Then also: $\widetilde{\mathbf{B}} \mathbf{Y}=\lambda \mathbf{Y}$, which can be written

$$
\left(\begin{array}{lll}
\beta_{00} & \beta_{01} & \beta_{02} \\
\beta_{10} & \beta_{11} & \beta_{12} \\
\beta_{20} & \beta_{21} & \beta_{22}
\end{array}\right)\left(\begin{array}{c}
y_{0} \\
y_{1} \\
1
\end{array}\right)=\left(\begin{array}{c}
\lambda y_{0} \\
\lambda y_{1} \\
\lambda
\end{array}\right),
$$

where each $\beta_{i j}$ is an integer. Then we have: $\beta_{20} y_{0}+\beta_{21} y_{1}+\beta_{22}=\lambda$. From now on, we suppose that $\lambda$ is a quadratic real number.

First Case: $\beta_{21}=0$. In this case, $y_{0}$ belongs to $\mathbb{Q}(\lambda)$. In addition, the same equality of matrices provides $\beta_{10} y_{0}+\beta_{11} y_{1}+\beta_{12}=\lambda y_{1}$, which can be 
written: $\beta_{10} y_{0}+\left(\beta_{11}-\lambda\right) y_{1}+\beta_{12}=0$. Then $y_{1}=\frac{\beta_{10} y_{0}+\beta_{12}}{\lambda-\beta_{11}}$ and $y_{1}$ also belongs to $\mathbb{Q}(\lambda)$.

Second Case: $\beta_{21} \neq 0$. Then: $y_{1}=\gamma_{0} y_{0}+\gamma_{1} \lambda+\gamma_{2}$, with $\gamma_{0}, \gamma_{1}, \gamma_{2}$ rationals. The same equality of matrices provides $\beta_{00} y_{0}+\beta_{01} y_{1}+\beta_{02}=\lambda y_{0}$, or $\left(\beta_{00}+\beta_{01} \gamma_{0}-\lambda\right) y_{0}+\beta_{01} \gamma_{1} \lambda+\gamma_{2}+\beta_{02}=0$. Then $y_{0}=\frac{\beta_{01} \gamma_{1} \lambda+\gamma_{2}+\beta_{02}}{\lambda-\beta_{00}-\beta_{01} \gamma_{0}}$. Then, in both cases, both $y_{0}$ and $y_{1}$ belong to $\mathbb{Q}(\lambda)$, which is a vectorial space with dimension 2 over $\mathbb{Q}$. Then the three numbers $y_{0}, y_{1}$ and 1 are rationally dependent. So are therefore the three numbers $x_{0}^{(s)}, x_{1}^{(s)}, x_{2}^{(s)}$, and then the three numbers $x_{0}=x_{0}^{(0)}, x_{1}=x_{1}^{(0)}, x_{2}=x_{2}^{(0)}$, because $\mathbf{X}^{(0)}=\mathbf{B}^{(s)} \mathbf{X}^{(s)}$. This contradicts our hypothesis. Then $\lambda$ cannot be a quadratic real number.

Lemma 4. (Converse Statement in Lagrange Theorem). Let $\mathbf{X}=$ $\mathrm{T}\left(x_{0}, x_{1}, x_{2}\right)$ be any rationally independent triplet of real numbers, with $0<x_{0}<x_{1}<x_{2}$. Let's suppose that the Smallest Vector Algorithm applied on the triplet $\mathbf{X}$ makes a "loop" i.e. that $\mathbf{X}^{(s+p)}=\lambda \mathbf{X}^{(s)}$ with $p>0$. Then $\lambda$ is an algebraic integer of degree 3, and a unit. The minimal polynomial of $\lambda$ can be easily deduced from the relation $\mathbf{X}^{(s+p)}=\lambda \mathbf{X}^{(s)}$ as also the expressions of $\frac{x_{0}}{x_{2}}$ and $\frac{x_{1}}{x_{2}}$ as rational fractions of $\lambda$.

Proof. By hypothesis, we have: $\mathbf{X}^{(s+p)}=\lambda \mathbf{X}^{(s)}$, or equivalently

$$
\left(\mathbf{B}^{(s+p)}\right)^{-1} \mathbf{X}=\lambda\left(\mathbf{B}^{(s)}\right)^{-1} \mathbf{X} \text { or }\left(\mathbf{B}^{(s+p)}\right)^{-1}\left(\mathbf{B}^{(s)}\right) \mathbf{X}^{(s)}=\lambda \mathbf{X}^{(s)} .
$$

Let's denote: $\widetilde{\mathbf{B}}:=\left(\mathbf{B}^{(s+p)}\right)^{-1}\left(\mathbf{B}^{(s)}\right)$. Then: $\widetilde{\mathbf{B}} \mathbf{X}^{(s)}=\lambda \mathbf{X}^{(s)}$. Let $F(\xi)$ be the polynomial: $F(\xi)=\operatorname{det}(\widetilde{\mathbf{B}}-\xi \mathbf{I})$, we have: $F(\lambda)=0$. But $\widetilde{\mathbf{B}}$ is an integer matrix with determinant \pm 1 ; then we have a relation of the shape: $\lambda^{3}+m \lambda^{2}+n \lambda \pm 1=0$, with $m$ and $n$ natural integers.

Then $\lambda$ is an algebraic integer and a unit. Then, if $\lambda$ is a rational number, it has to be: $\lambda= \pm 1$. But that's impossible because we should have $\mathbf{X}^{(s+p)}=$ $\pm \mathbf{X}^{(s)}$, which is impossible by the subtractive form of the recursive relation on the sequence $\left(\mathbf{X}^{(s)}\right)$. By the previous Lemma $\lambda$ cannot be a root of a second degree polynomial. Then $F(\xi)$ is the minimal polynomial of $\lambda$ over $\mathbb{Q}$, and $\lambda$ is a cubic algebraic integer. Its norm is 1 , and the relation $\lambda\left(\lambda^{2}+m \lambda+n\right)=\mp 1$ shows that $\lambda$ is a unit.

Now, we have to solve the equation $(\widetilde{\mathbf{B}}-\lambda \mathbf{I}) \mathbf{X}^{(s)}=0$, or $(\widetilde{\mathbf{B}}-\lambda \mathbf{I}) \mathbf{Y}=$ $\mathbf{0}, \mathbf{Y}$ being the unknown vector, with the classic method of linear algebra. Because $\mathbf{Y}=\left(\mathbf{B}^{(s)}\right)^{-1} \mathbf{X}$, the triplet $\mathbf{Y}$ is rationally independent, then $y_{3} \neq 0$. If we just want to obtain one vector solution, we may even suppose that $y_{3}=1$. Let $\mathbf{A}$ be the matrix $(\widetilde{\mathbf{B}}-\lambda \mathbf{I})$ without its third row, let $\mathbf{a}_{2}$ be 
the third column of the matrix $\mathbf{A}$, and let $\mathbf{A}^{\prime}$ be the matrix $\mathbf{A}$ without its third column. Let also $\mathbf{Y}^{\prime}$ be the vector $\mathbf{Y}$ without its third coordinate. With block submatrices, we have to solve in $\mathbf{Y}^{\prime}$ the equation: $\left[\begin{array}{ll}\mathbf{A}^{\prime} & ; \mathbf{a}_{2}\end{array}\right] \times\left[\begin{array}{c}\mathbf{Y}^{\prime} \\ 1\end{array}\right]=$ 0. This gives $\mathbf{A}^{\prime} \times \mathbf{Y}^{\prime}+\mathbf{a}_{2} \times 1=\mathbf{0}$, or $\mathbf{Y}^{\prime}=-\left(\mathbf{A}^{\prime}\right)^{-1} \mathbf{a}_{2}$. We know that $\operatorname{det}\left(\mathbf{A}^{\prime}\right) \neq 0$, because $F(\xi)$ is the minimal polynomial of $\lambda$. Then we have $\mathbf{X}^{(s)}=\left[\begin{array}{c}-\alpha\left(\mathbf{A}^{\prime}\right)^{-1} \mathbf{a}_{2} \\ \alpha\end{array}\right]$, for some $\alpha$, and then: $\mathbf{X}=\mathbf{B}^{(s)} \mathbf{X}^{(s)}=\alpha \mathbf{B}^{(s)} \times$ $\left[\begin{array}{c}-\left(\mathbf{A}^{\prime}\right)^{-1} \mathbf{a}_{2} \\ 1\end{array}\right]$. Of course, $\mathbf{X}$ is defined up to a multiplicative coefficient. Note that $\mathbf{A}^{\prime}$ and $\mathbf{a}_{2}$ are rational fractions of the unit $\lambda$. Then the vector $\mathbf{Z}:=\left[\begin{array}{c}-\left(\mathbf{A}^{\prime}\right)^{-1} \mathbf{a}_{2} \\ 1\end{array}\right]$ can be also obtained as an expression of $\lambda$. Let $\widehat{\mathbf{b}}_{0}, \widehat{\mathbf{b}}_{1}$, $\widehat{\mathbf{b}}_{2}$ be the three rows of the matrix $\mathbf{B}^{(s)}$; then we have

$$
\left(\begin{array}{c}
x_{0} / \alpha \\
x_{1} / \alpha \\
x_{2} / \alpha
\end{array}\right)=\left(\begin{array}{c}
\widehat{\mathbf{b}}_{0} \\
\widehat{\mathbf{b}}_{1} \\
\widehat{\mathbf{b}}_{2}
\end{array}\right) \mathbf{Z}=\left(\begin{array}{c}
\widehat{\mathbf{b}}_{0} \mathbf{Z} \\
\widehat{\mathbf{b}}_{1} \mathbf{Z} \\
\widehat{\mathbf{b}}_{2} \mathbf{Z}
\end{array}\right) .
$$

Then $\frac{x_{0}}{x_{2}}=\frac{\widehat{\mathbf{b}}_{0} \mathbf{Z}}{\widehat{\mathbf{b}}_{2} \mathbf{Z}}$ and $\frac{x_{1}}{x_{2}}=\frac{\widehat{\mathbf{b}}_{1} \mathbf{Z}}{\widehat{\mathbf{b}}_{2} \mathbf{Z}}$ and we can express $\frac{x_{0}}{x_{2}}$ and $\frac{x_{1}}{x_{2}}$ as rational fractions of $\lambda$.

2.2. Demonstration of the Lagrange Property (particular case). In this subsection, we prove the Theorem on the Lagrange Property, admitting the Dirichlet Theorem which is proved in other section; we're doing this proof only in the special case $\mathbf{X}={ }^{\mathrm{T}}\left(1, \theta, \theta^{2}\right)$, with $\theta=\sqrt[3]{N}$, $N$ being an natural number, but not $\theta$. For the complete proof, see Section 5.. We're going to prove that the Smallest Vector Algorithm in this case makes a loop.

It is a basic result in Diophantine Approximation that there exists a real number $e>0$ such that for any non-null integer point $\mathbf{h}={ }^{\mathrm{T}}(m, n, p)$, the inequality $(\max (|m|,|n|,|p|))^{2} \times|\mathbf{h} \bullet \mathbf{X}|>e$ holds. See for instance [6] (Cassels), Theorem III, page 79, statement (2), which is much stronger. But in finite dimension, all the norms are equivalent. Then there exists $d>0$ such that for any non-null integer point $\mathbf{h}$, the inequality $\|\mathbf{h}\|^{2}|\mathbf{h} \bullet \mathbf{X}|>$ $d$ holds, id est $\|\mathbf{h}\|^{2}\left\|\mathbf{h}^{\prime \prime}\right\| \times\|\mathbf{X}\|>d$. Then, by our Dirichlet Property Theorem 2, which we admit temporarily, there exists an infinite set of integers $S$ such that:

$$
\sup _{s \in S}\left[\left(\max _{i=0,1,2}\left\|\mathbf{b}_{i}^{\prime(s)}\right\|\right)^{2}\left(\max _{i=0,1,2}\left\|\mathbf{b}_{i}^{\prime \prime(s)}\right\|\right)\right]=L<+\infty
$$


with in addition: $\lim _{s \rightarrow+\infty, s \in S}\left(\max _{i=0,1,2}\left\|\mathbf{b}_{i}^{\prime(s)}\right\|\right)=0$.

Let $s$ be any element of $S$, and let $\left(\mathbf{b}_{0}^{(s)}, \mathbf{b}_{1}^{(s)}, \mathbf{b}_{2}^{(s)}\right)$ be the column vectors of $\left(\mathbf{B}^{(s)}\right)$. We choose one of those three vectors, say $\mathbf{b}_{0}^{(s)}$, which will be more simply denoted: $\mathbf{b}^{(s)}=\mathbf{b}_{0}^{(s)}$. Let's define its coordinates:

$$
\mathbf{b}^{(s)}=\mathrm{T}\left(b_{x}^{(s)}, b_{y}^{(s)}, b_{z}^{(s)}\right) .
$$

From now on and for a while, we may omit the indices ${ }^{(s)}$.

Notation 1. The notation $\mathbf{M}_{\mathbf{b}}^{(s)}=\mathbf{M}_{\mathbf{b}}$ or $\mathbf{M}\left[\mathbf{b}^{(s)}\right]$ will denote the integer matrix: $\mathbf{M}_{\mathbf{b}}=\mathbf{M}\left[\mathbf{b}^{(s)}\right]=\left(\begin{array}{ccc}b_{z} & b_{y} & b_{x} \\ N \cdot b_{x} & b_{z} & b_{y} \\ N \cdot b_{y} & N \cdot b_{x} & b_{z}\end{array}\right)$, which has the interesting property: $\mathbf{M}_{\mathbf{b}} \mathbf{X}=\mathbf{M}_{\mathbf{b}}\left(\begin{array}{l}1 \\ \theta \\ \theta^{2}\end{array}\right)=\left(b_{z}+b_{y} \theta+b_{x} \theta^{2}\right)\left(\begin{array}{l}1 \\ \theta \\ \theta^{2}\end{array}\right)$. Let's denote by $\lambda_{\mathbf{b}}$ or $\lambda\left[\mathbf{b}^{(s)}\right]$ the following element of $\mathbb{Z}[\theta]$ :

$$
\lambda_{\mathbf{b}}:=\left(b_{z}+b_{y} \theta+b_{x} \theta^{2}\right) .
$$

Then we have: $\mathbf{M}_{\mathbf{b}} \mathbf{X}=\lambda_{\mathbf{b}} \mathbf{X}$; i.e. $\lambda_{\mathbf{b}}$ is an eigenvalue of $\mathbf{M}_{\mathbf{b}}$ with eigenvector $\mathbf{X}$.

We consider the sequence of the matrices $\boldsymbol{\Pi}^{(s)}=\boldsymbol{\Pi}={ }^{\mathrm{T}} \mathbf{M}_{\mathbf{b}} \mathbf{G}$. Let $\left(\mathbf{b}^{\# \#}, \mathbf{b}^{\#}, \mathbf{b}\right)$ be the three column vectors of $\mathbf{M}_{\mathbf{b}}$. Then

$$
\mathbf{\Pi}={ }^{\mathrm{T}} \mathbf{M}_{\mathbf{b}} \mathbf{G}=\left(\begin{array}{ccc}
\mathbf{b}^{\# \#} \bullet \mathbf{g}_{0} & \mathbf{b}^{\# \#} \bullet \mathbf{g}_{1} & \mathbf{b}^{\# \#} \bullet \mathbf{g}_{2} \\
\mathbf{b}^{\#} \bullet \mathbf{g}_{0} & \mathbf{b}^{\#} \bullet \mathbf{g}_{1} & \mathbf{b}^{\#} \bullet \mathbf{g}_{2} \\
\mathbf{b} \bullet \mathbf{g}_{0} & \mathbf{b} \bullet \mathbf{g}_{1} & \mathbf{b} \bullet \mathbf{g}_{1}
\end{array}\right):=\left(\pi_{i, j}\right)
$$

$i=0,1,2 ; j=0,1,2$.

Lemma 5 (Main Lemma for Lagrange). The matrices $\Pi^{(s)}$ are bounded independently from $s$.

Proof. We have to find an upper bound for each of the $\left|\pi_{i, j}\right|, i=0,1,2$; $j=0,1,2$. Let's consider first the $\left|\mathbf{b}^{\#} \bullet \mathbf{g}_{i}\right|$. We have:

$$
\mathbf{b}^{\#}=\mathbf{Q}^{\#}\left(\begin{array}{c}
b_{x} \\
b_{y} \\
b_{z}
\end{array}\right)=\mathbf{Q}^{\#} \mathbf{b}, \text { with } \mathbf{Q}^{\#}=\left(\begin{array}{ccc}
0 & 1 & 0 \\
0 & 0 & 1 \\
N & 0 & 0
\end{array}\right)
$$

We also have $\mathbf{Q}^{\#} \mathbf{X}=\theta \mathbf{X}$. Let $\mathbf{n}$ be: $\mathbf{n}=\frac{\mathbf{X}}{\|\mathbf{X}\|}$. Then also $\mathbf{Q}^{\#} \mathbf{n}=\theta \mathbf{n}$. 
We have, with always the same kind of notations, $\mathbf{b}^{\#}=\mathbf{b}^{\# \prime}+\mathbf{b}^{\# \prime \prime}$,

$$
\mathbf{b}^{\#} \cdot \mathbf{g}_{i}=\left(\mathbf{b}^{\# \prime}+\mathbf{b}^{\# \prime \prime}\right) \bullet\left(\mathbf{g}_{i}^{\prime}+\mathbf{g}_{i}^{\prime \prime}\right)=\mathbf{b}^{\# \prime} \bullet \mathbf{g}_{i}^{\prime}+\mathbf{b}^{\# \prime \prime} \bullet \mathbf{g}_{i}^{\prime \prime},
$$

with $\mathbf{g}_{i}^{\prime}= \pm\left(\mathbf{b}_{j}^{\prime \prime} \wedge \mathbf{b}_{k}^{\prime}+\mathbf{b}_{j}^{\prime} \wedge \mathbf{b}_{k}^{\prime \prime}\right) ; \mathbf{g}_{i}^{\prime \prime}= \pm \mathbf{b}_{j}^{\prime} \wedge \mathbf{b}_{k}^{\prime}$. Then:

$$
\left|\mathbf{b}^{\#} \bullet \mathbf{g}_{i}\right| \leq\left\|\mathbf{b}^{\# \prime}\right\|\left\|\mathbf{b}^{\prime \prime}{ }_{j}\right\|\left\|\mathbf{b}_{k}^{\prime}\right\|+\left\|\mathbf{b}^{\# \prime}\right\|\left\|\mathbf{b}_{j}^{\prime}\right\|\left\|\mathbf{b}^{\prime \prime}{ }_{k}\right\|+\left\|\mathbf{b}^{\# \prime \prime}\right\|\left\|\mathbf{b}_{j}^{\prime}\right\|\left\|\mathbf{b}_{k}^{\prime}\right\| .
$$

Let's denote: $\beta^{\prime}=\max _{i=0,1,2}\left\|\mathbf{b}_{i}^{\prime}\right\|$ and $\beta^{\prime \prime}=\max _{i=0,1,2}\left\|\mathbf{b}_{i}^{\prime \prime}\right\|$, so that: $\left(\beta^{\prime}\right)^{2} \beta^{\prime \prime} \leq L$. Then:

$$
\left|\mathbf{b}^{\#} \cdot \mathbf{g}_{i}\right| \leq\left\|\mathbf{b}^{\# \prime}\right\| \beta^{\prime \prime} \beta^{\prime}+\left\|\mathbf{b}^{\# \prime}\right\| \beta^{\prime} \beta^{\prime \prime}+\left\|\mathbf{b}^{\# \prime \prime}\right\|\left(\beta^{\prime}\right)^{2} .
$$

But we also have: $\mathbf{b}^{\#}=\mathbf{Q}^{\#} \mathbf{b}=\mathbf{Q}^{\#}\left(\mathbf{b}_{0}^{\prime}+\left\|\mathbf{b}_{0}^{\prime \prime}\right\| \mathbf{n}\right)=\mathbf{Q}^{\#} \mathbf{b}_{0}^{\prime}+\left\|\mathbf{b}_{0}^{\prime \prime}\right\| \theta \mathbf{n}$. This proves first that the distance $\left\|\mathbf{b}^{\# \prime}\right\|$ between $\mathbf{b}^{\#}$ and $\mathbb{D}$ is less than $\left\|\mathbf{Q}^{\#} \mathbf{b}_{0}^{\prime}\right\|$ :

$$
\left\|\mathbf{b}^{\# \prime}\right\| \leq\left\|\mathbf{Q}^{\#} \mathbf{b}_{0}^{\prime}\right\| \leq\left\|\mathbf{Q}^{\#}\right\| \times\left\|\mathbf{b}_{0}^{\prime}\right\| \leq\left\|\mathbf{Q}^{\#}\right\| \beta^{\prime}
$$

(this using the norm of the matrix). Furthermore, we have the following equality: $\mathbf{b}^{\# \prime}+\mathbf{b}^{\# \prime \prime}=\mathbf{b}^{\#}=\mathbf{Q}^{\#} \mathbf{b}_{0}^{\prime}+\left\|\mathbf{b}_{0}^{\prime \prime}\right\| \theta \mathbf{n}$, and we deduce:

$$
\mathbf{b}^{\# \prime \prime}=\left\|\mathbf{b}_{0}^{\prime \prime}\right\| \theta \mathbf{n}+\mathbf{Q}^{\#} \mathbf{b}_{0}^{\prime}-\mathbf{b}^{\# \prime} \text {. }
$$

Then:

$$
\left\|\mathbf{b}^{\# \prime \prime}\right\| \leq\left\|\mathbf{b}_{0}^{\prime \prime}\right\| \theta+2\left\|\mathbf{Q}^{\#}\right\|\left\|\mathbf{b}_{0}^{\prime}\right\| \leq \beta^{\prime \prime} \theta+2\left\|\mathbf{Q}^{\#}\right\| \beta^{\prime}
$$

Putting 2.2 and 2.3 in 2.1 , we obtain:

$$
\left|\mathbf{b}^{\#(s)} \bullet \mathbf{g}_{i}^{(s)}\right| \leq\left(\beta^{\prime(s)}\right)^{2} \beta^{\prime \prime(s)}\left(2\left\|\mathbf{Q}^{\#}\right\|+\theta\right)+2\left\|\mathbf{Q}^{\#}\right\|\left(\beta^{\prime(s)}\right)^{3}
$$

and then:

$$
\left|\mathbf{b}^{\#(s)} \bullet \mathbf{g}_{i}^{(s)}\right| \leq L\left(2\left\|\mathbf{Q}^{\#}\right\|+\theta\right)+2\left\|\mathbf{Q}^{\#}\right\|\left(\beta^{\prime(s)}\right)^{3} .
$$

The limit of the last term is 0 , by the $\mathbf{c}$ ) of the Dirichlet Properties Theorem. Then the set of the $\left|\mathbf{b}^{\#(s)} \bullet \mathbf{g}_{i}^{(s)}\right|$, with $s$ in $S$, is bounded. By a similar demonstration, we prove that the numbers $\left|\mathbf{b}^{\# \#(s)} \bullet \mathbf{g}_{i}^{(s)}\right|$ are also bounded and so are, in an obvious way, the numbers $\left|\mathbf{b}_{0}^{(s)} \bullet \mathbf{g}_{i}^{(s)}\right|$. Then the set of the $\Pi^{(s)}$ is bounded, and the Lemma is proved.

With this Lemma, the demonstration of the Lagrange result is easy. 
Proof of the Lagrange Result. The set of the $\Pi^{(s)}$ with $s$ in $S$ is bounded; but these matrices have integral coefficients. Then the number of all the $\boldsymbol{\Pi}^{(s)}$ is finite. Then there exist $s$ and $t$, with $s<t$, such that $\boldsymbol{\Pi}^{(t)}=\boldsymbol{\Pi}^{(s)}$. This means: ${ }^{\mathrm{T}} \mathbf{M}\left[\mathbf{b}^{(t)}\right] \times \mathbf{G}^{(t)}={ }^{\mathrm{T}} \mathbf{M}\left[\mathbf{b}^{(s)}\right] \times \mathbf{G}^{(s)}$. By transposition:

$$
\left(\mathbf{B}^{(t)}\right)^{-1} \times \mathbf{M}\left[\mathbf{b}^{(t)}\right]=\left(\mathbf{B}^{(s)}\right)^{-1} \times \mathbf{M}\left[\mathbf{b}^{(s)}\right] .
$$

We apply that to the column vector $\mathbf{X}$; we obtain

$$
\left(\mathbf{B}^{(t)}\right)^{-1} \times \mathbf{M}\left[\mathbf{b}^{(t)}\right] \times \mathbf{X}=\left(\mathbf{B}^{(s)}\right)^{-1} \times \mathbf{M}\left[\mathbf{b}^{(s)}\right] \times \mathbf{X}
$$

then, by "eigenvalue", see Notation above:

$$
\left(\mathbf{B}^{(t)}\right)^{-1} \times\left(\lambda\left[\mathbf{b}^{(t)}\right] \cdot \mathbf{X}\right)=\left(\mathbf{B}^{(s)}\right)^{-1} \times\left(\lambda\left[\mathbf{b}^{(s)}\right] \cdot \mathbf{X}\right) .
$$

We recall that $\lambda\left[\mathbf{b}^{(s)}\right]$ is a real number in $\mathbb{Z}[\theta]$. Then

$$
\left(\mathbf{B}^{(t)}\right)^{-1} \mathbf{X}=\frac{\lambda\left[\mathbf{b}^{(s)}\right]}{\lambda\left[\mathbf{b}^{(t)}\right]} \cdot\left(\mathbf{B}^{(s)}\right)^{-1} \mathbf{X} .
$$

This reads $\mathbf{X}^{(t)}=\lambda \mathbf{X}^{(s)}$, with $\lambda=\frac{\lambda\left[\mathbf{b}^{(s)}\right]}{\lambda\left[\mathbf{b}^{(t)}\right]}$, and the first part of the Theorem is obtained.

We have already proved the Second Part of the Theorem, by Lemma 4 of the previous subsection. With this Second Part, we also obtain the final assertions of the First Part.

In particular, $x_{0}, x_{1}, x_{2}$, are rationally independent, then so are also $\frac{x_{0}}{x_{2}}$, $\frac{x_{1}}{x_{2}}$ and 1 . Then $\left(\frac{x_{0}}{x_{2}}, \frac{x_{1}}{x_{2}}, 1\right)$ is a basis of $\mathbb{Q}(\rho)$ over $\mathbb{Q}$. But we know by the Lemma 4 that $\frac{x_{0}}{x_{2}}$ and $\frac{x_{1}}{x_{2}}$ are rational fractions of $\lambda$, therefore they belong to $\mathbb{Q}(\lambda)$ and then $\mathbb{Q}(\rho)=\mathbb{Q}(\lambda)$.

\section{From the Geometrical Property to the Dirichlet Properties}

In this section, the Geometrical Theorem is admitted, and we prove the Theorem 2 on Dirichlet Properties.

\subsection{Proof of Part a) of Theorem 2.}

Lemma 6 (Prism Lemma). Let $\mathbf{g}_{0}^{(s)}, \mathbf{g}_{1}^{(s)}, \mathbf{g}_{2}^{(s)}$ be the column vectors of the matrix $\mathbf{G}^{(s)}$ generated at the s-th stage by the Smallest Vector Algorithm. Let the sets $\mathbf{H}^{\prime(s)}$ be the convex hulls:

$$
\mathbf{H}^{\prime(s)}=\operatorname{CONV}\left(\mathbf{g}_{0}^{\prime(s)}, \mathbf{g}_{1}^{\prime(s)}, \mathbf{g}_{2}^{\prime(s)},-\mathbf{g}_{0}^{\prime(s)},-\mathbf{g}_{1}^{\prime(s)},-\mathbf{g}_{2}^{\prime(s)}\right) \text { in } \mathbb{P} .
$$

We shall omit the indices ${ }^{(s)}$. Let $\mathbf{H}$ be the prism: $\mathbf{H}:=\mathbf{H}^{\prime}+\mathbb{D}$. Then, with the usual notation $\mathbf{h}^{\prime \prime}$ for the orthogonal projection of $\mathbf{h}$ on $\mathbb{D}$ :

-For each non zero integer point $\mathbf{h}$ in $\mathbf{H},\left\|\mathbf{h}^{\prime \prime}\right\| \geq\left\|\mathbf{g}_{0}^{\prime \prime}\right\|$ holds. 
-For each integer point $\mathbf{h}$ in $\mathbf{H}$ which is not of the form $n_{0} \mathbf{g}_{0}$, with $n_{0}$ integer, $\left\|\mathbf{h}^{\prime \prime}\right\| \geq\left\|\mathbf{g}_{1}^{\prime \prime}\right\|$ holds.

-For each integer point $\mathbf{h}$ in $\mathbf{H}$ which is not of the form $n_{0} \mathbf{g}_{0}+n_{1} \mathbf{g}_{1}$, with $n_{0}$ and $n_{1}$ integers, $\left\|\mathbf{h}^{\prime \prime}\right\| \geq\left\|\mathbf{g}_{2}^{\prime \prime}\right\|$ holds.

- This implies that, if $\left(\mathbf{h}_{0}, \mathbf{h}_{1}, \mathbf{h}_{2}\right)$ is a free triplet of integer points in $\mathbf{H}$, then $\max _{i=0,1,2}\left(\left\|\mathbf{h}_{i}^{\prime \prime}\right\|\right) \geq \max _{i=0,1,2}\left(\left\|\mathbf{g}_{i}^{\prime \prime}\right\|\right)=\left\|\mathbf{g}_{2}^{\prime \prime}\right\|$.

Proof. Let $\mathbf{h}$ be any non-null integer vector in $\mathbf{H}$. Since $\operatorname{det}\left(\mathbf{G}^{(s)}\right)= \pm 1$, there exist three relative integers $n_{0}, n_{1}, n_{2}$ such that $\mathbf{h}=n_{0} \mathbf{g}_{0}+n_{1} \mathbf{g}_{1}+n_{2} \mathbf{g}_{2}$. Let $\mathbf{h}^{\prime}$ be the orthogonal projection of $\mathbf{h}$ on $\mathbb{P}$. We have $\mathbf{h}^{\prime}=n_{0} \mathbf{g}_{0}^{\prime}+n_{1} \mathbf{g}_{1}^{\prime}+$ $n_{2} \mathbf{g}_{2}^{\prime}$ and also $\mathbf{h}^{\prime} \in \mathbf{H}^{\prime}$, which is the convex hull of six points. But an easy geometrical study shows that, in the plane, if $\mathbf{p}$ is in the convex hulls of six points, it has to be in the convex hull of three among these six points. In this proof, "positive" will mean: " $\geq 0$ ", and not " $>0 "$ ".

Then $\mathbf{h}^{\prime}$ is the positive barycenter of three points among $\mathbf{g}_{0}^{\prime}, \mathbf{g}_{1}^{\prime}, \mathbf{g}_{2}^{\prime},-\mathbf{g}_{0}^{\prime}$, $-\mathbf{g}_{1}^{\prime},-\mathbf{g}_{2}^{\prime}$. This means that $\mathbf{h}^{\prime}$ is the positive barycenter of three points of the shape $\varepsilon_{0} \mathbf{g}_{0}^{\prime}, \varepsilon_{1} \mathbf{g}_{1}^{\prime}, \varepsilon_{2} \mathbf{g}_{2}^{\prime}$, with $\varepsilon_{i} \in\{-1,1\}$. Then there exist positive real numbers $y_{0}, y_{1}, y_{2}$ such that $y_{0}+y_{1}+y_{2}=1$ and such that

$$
\mathbf{h}^{\prime}=y_{0} \varepsilon_{0} \mathbf{g}_{0}^{\prime}+y_{1} \varepsilon_{1} \mathbf{g}_{1}^{\prime}+y_{2} \varepsilon_{2} \mathbf{g}_{2}^{\prime}=n_{0} \mathbf{g}_{0}^{\prime}+n \mathbf{g}_{1}^{\prime}+n_{2} \mathbf{g}_{2}^{\prime} .
$$

Then $\left(n_{0}-y_{0} \varepsilon_{0}\right) \mathbf{g}_{0}^{\prime}+\left(n_{1}-y_{1} \varepsilon_{1}\right) \mathbf{g}_{1}^{\prime}+\left(n_{2}-y_{2} \varepsilon_{2}\right) \mathbf{g}_{2}^{\prime}=\mathbf{0}$. But we have also $\|\mathbf{X}\|\left\|\mathbf{b}_{0}^{\prime \prime(s)}\right\| \mathbf{g}_{0}^{(s)}+\|\mathbf{X}\|\left\|\mathbf{b}_{1}^{\prime \prime(s)}\right\| \mathbf{g}_{1}^{(s)}+\|\mathbf{X}\|\left\|\mathbf{b}_{2}^{\prime \prime(s)}\right\| \mathbf{g}_{2}^{(s)}=\mathbf{X}$, like in the first Lemma of the Section 2, and then, with $z_{i}=\|\mathbf{X}\|\left\|\mathbf{b}_{0}^{\prime \prime(s)}\right\|$, we have:

$$
z_{0} \mathbf{g}_{0}^{\prime}+z_{1} \mathbf{g}_{1}^{\prime}+z_{2} \mathbf{g}_{2}^{\prime}=\mathbf{0}
$$

with $z_{i}>0$, by orthogonal projection on $\mathbb{P}$. By the uniqueness of the barycentrical coordinates, up to a multiplicative coefficient, we get: for some real number $\lambda$,

$$
n_{0}-y_{0} \varepsilon_{0}=\lambda z_{0} ; n_{1}-y_{1} \varepsilon_{1}=\lambda z_{1} ; n_{2}-y_{2} \varepsilon_{2}=\lambda z_{2} .
$$

Then $n_{0}-y_{0} \varepsilon_{0}, n_{1}-y_{1} \varepsilon_{1}, n_{2}-y_{2} \varepsilon_{2}$ have the same sign (0 has both signs). Without loss of generality, this sign may be supposed to be positive. Then we have $n_{0} \geq y_{0} \varepsilon_{0} \geq-1, n_{1} \geq y_{1} \varepsilon_{1} \geq-1, n_{2} \geq y_{2} \varepsilon_{2} \geq-1$.

- If for every $i=1,2,3$, we have $n_{i}>-1 \mathrm{~h}$, then $n_{0}, n_{1}, n_{2}$ have the same sign.

- If now, for some $i$, we have $n_{i}=y_{i} \varepsilon_{i}=-1$, then $y_{i}=1$, and $y_{j}=y_{k}=0$, with $\{i, j, k\}=\{0,1,2\}$. Moreover, $\lambda=0$, and then $n_{i}=-1, n_{j}=y_{j} \varepsilon_{j}=0$, $n_{k}=y_{k} \varepsilon_{k}=0$. Then, in every case, $n_{0}, n_{1}, n_{2}$ have the same sign. Then $\left\|\mathbf{h}^{\prime \prime}\right\|=\left\|n_{0} \mathbf{g}_{0}^{\prime \prime}+n_{1} \mathbf{g}_{1}^{\prime \prime}+n_{2} \mathbf{g}_{0}^{\prime \prime}\right\|=\left|n_{0}\right|\left\|\mathbf{g}_{0}^{\prime \prime}\right\|+\left|n_{1}\right|\left\|\mathbf{g}_{1}^{\prime \prime}\right\|+\left|n_{2}\right|\left\|\mathbf{g}_{2}^{\prime \prime}\right\|$, and the conclusions of the theorem become obvious.

Let's notice that the Prism Lemma shows that in some way, our algorithm gives best integer approximations of the plane on $\mathbb{P}$. In fact, each $\mathbf{g}_{0}^{(s)}$ is a 
best approximation, and so are, after the $n_{0} \mathbf{g}_{0}^{(s)}$, the vector $\mathbf{g}_{1}^{(s)}$ and, after the vectors $n_{0} \mathbf{g}_{0}^{(s)}+n_{1} \mathbf{g}_{1}^{(s)}$, the vector $\mathbf{g}_{2}^{(s)}$. The vectors $\mathbf{g}_{0}^{(s)}, \mathbf{g}_{1}^{(s)}, \mathbf{g}_{2}^{(s)}$ are not necessarily successive best approximations with disks of the euclidean norm in $\mathbb{P}$, but so are they for the "hexagon" (which can be a parallelogram) $\mathbf{H}^{\prime(s)}$, which depends on $\left(\mathbf{g}_{0}^{(s)}, \mathbf{g}_{1}^{(s)}, \mathbf{g}_{2}^{(s)}\right)$ themselves.

Proof. Let's now prove the assertion a) of Theorem 2.

We recall that, by convention, $\left\|\mathbf{g}_{0}^{\prime \prime(s+1)}\right\| \leq\left\|\mathbf{g}_{1}^{\prime \prime(s+1)}\right\| \leq\left\|\mathbf{g}_{2}^{\prime \prime(s+1)}\right\|$ and we denote by $\left(\mathbf{g}_{\mathrm{I}}^{(s)}, \mathbf{g}_{\mathrm{II}}^{(s)}, \mathbf{g}_{\mathrm{III}}^{(s)}\right)$ the permutation of $\left(\mathbf{g}_{0}^{(s)}, \mathbf{g}_{1}^{(s)}, \mathbf{g}_{2}^{(s)}\right)$ such that $\left\|\mathbf{g}_{\mathrm{I}}^{\prime(s)}\right\| \leq\left\|\mathbf{g}_{\mathrm{II}}^{\prime(s)}\right\| \leq\left\|\mathbf{g}_{\mathrm{III}}^{\prime(s)}\right\|$. We admit the Geometrical Theorem, namely that there exists an infinite set $S$ of natural integers such that:

$$
\sup _{s \in S} \frac{\left\|\mathbf{g}_{\mathrm{III}}^{(s)}\right\|}{\rho^{(s)}}=L<+\infty \text {. }
$$

This will be proved in the next section. For any $s \in S$, we consider the cylinder with center at $\mathbf{0}$, with basis in $\mathbb{P}$, radius $\rho^{(s)}$, and height $\frac{8}{\pi\left(\rho^{(s)}\right)^{2}}$, namely:

$$
\Gamma^{(s)}=\operatorname{Disk}^{\prime}\left(\rho^{(s)}\right)+\operatorname{Disk}^{\prime \prime}\left(\frac{4}{\pi\left(\rho^{(s)}\right)^{2}}\right)
$$

(the second disk being in $\mathbb{D}$, and being a segment). The volume of $\Gamma^{(s)}$ is 8. Then, by Minkowski's first Theorem, there is an integer point $\mathbf{h} \neq \mathbf{0}$ in $\Gamma^{(s)}$. For this theorem, see for instance, [6](Cassels), Theorem IV, page 154. Then we have, by the Prism Lemma for the second inequality,

$$
\left\|\mathbf{g}_{\text {III }}^{\prime(s)}\right\|^{2}\left\|\mathbf{g}_{0}^{\prime \prime(s)}\right\| \leq L^{2}\left(\rho^{(s)}\right)^{2}\left\|\mathbf{g}_{0}^{\prime \prime(s)}\right\| \leq L^{2}\left(\rho^{(s)}\right)^{2}\left\|\mathbf{h}^{\prime \prime}\right\| \leq \frac{4 L^{2}}{\pi}
$$

or $\left\|\mathbf{g}_{\mathrm{III}}^{\prime(s)}\right\|^{2}\left\|\mathbf{g}_{0}^{\prime \prime(s)}\right\| \leq M$ a constant, and the main statement of a) is proved. This last result, with the help of the Lemma 2 of Section 2, namely $\lim _{s \rightarrow+\infty, s \in S}\left\|\mathbf{g}_{\mathrm{III}}^{\prime(s)}\right\|=+\infty$, implies the last statement of a), id est

$$
\lim _{s \rightarrow+\infty, s \in S}\left\|\mathbf{g}_{0}^{\prime \prime(s)}\right\|=0 .
$$

3.2. Demonstration of assertions b) and c) in Theorem 2. We shall need some well known results in Diophantine Approximation or Geometry of Numbers.

Lemma 7 (Transference Theorem in $\operatorname{dim} 2$ ). Let $1, \alpha, \beta$ be three rationally independent real numbers, let $\mathbf{X}$ be: $\mathbf{X}={ }^{\mathrm{T}}(1, \alpha, \beta)$ and let $\mathbb{D}$ be $\mathbb{D}=\mathbb{R} \mathbf{X}$. 
Let $\mathbf{h}:=(m, n, p)$ be an integral point in $\mathbb{Z}^{3} \backslash\{\mathbf{0}\}$. The following six assertions are equivalent:
(a) $\inf _{\mathbf{h}=(m, n, p) \text { with }(n, p) \neq(0,0)}|\mathbf{X} \bullet \mathbf{h}| \times[\max (|n|,|p|)]^{2}>0$
(b) $\inf _{\mathbf{h}=(m, n, p) \text { with } m \neq 0}|m| \times[\max (|\alpha m-n|,|\beta m-p|)]^{2}>0$
$\left(\boldsymbol{a}^{\prime}\right) \inf _{\mathbf{h}=(m, n, p) \in \mathbb{Z}^{3} \backslash\{\mathbf{0}\}}|\mathbf{X} \bullet \mathbf{h}| \times[\max (|m|,|n|,|p|)]^{2}>0$
$\left(\boldsymbol{b}^{\prime}\right) \inf _{\mathbf{h}=(m, n, p) \in \mathbb{Z}^{3} \backslash\{\mathbf{0}\}} \max (|m|,|n|,|p|) \times[\max (|\alpha m-n|,|\beta m-p|)]^{2}>0$
(A) $\inf _{\mathbf{h} \in \mathbb{Z}^{3} \backslash\{\mathbf{0}\}}|\mathbf{X} \bullet \mathbf{h}| \times\|\mathbf{h}\|^{2}>0$
(B) $\inf _{\mathbf{h}=(m, n, p) \in \mathbb{Z}^{3} \backslash\{\mathbf{0}\}}\|\mathbf{h}\| \times\left((\alpha m-n)^{2}+(\beta m-p)^{2}\right)>0$

Proof. The equivalence of (a) with (b) is a classical result. See for instance: [6] (Cassels), Theorem II and Corollary. The other equivalences are also classical and easy.

Lemma 8 (Transference Theorem, geometrical point of view). Let $1, \alpha, \beta$ be three rationally independent real numbers, let $\mathbf{X}$ be: $\mathbf{X}={ }^{\mathrm{T}}(1, \alpha, \beta)$ and let $\mathbb{D}$ be $\mathbb{D}=\mathbb{R} \mathbf{X}$. As usual, let $\mathbf{h}^{\prime}$ and $\mathbf{h}^{\prime \prime}$ be the orthogonal projections of $\mathbf{h}$ on $\mathbb{D}$ and $\mathbb{P}=\mathbb{D}^{\perp}$. The following three assertions are equivalent, and also are equivalent to assertions $(A)$ and $(B)$ of the previous Lemma.

(C) $\inf _{\mathbf{h} \in \mathbb{Z}^{3} \backslash\{\mathbf{0}\}}\left\|\mathbf{h}^{\prime \prime}\right\| \times\|\mathbf{h}\|^{2}>0$

(D) $\inf _{\mathbf{h} \in \mathbb{Z}^{3} \backslash\{\mathbf{0}\}}\|\mathbf{h}\| \times\left\|\mathbf{h}^{\prime}\right\|^{2}>0$

(E) $\inf _{\mathbf{h} \in \mathbb{Z}^{3} \backslash\{\mathbf{0}\}}\left\|\mathbf{h}^{\prime \prime}\right\| \times\left\|\mathbf{h}^{\prime}\right\|^{2}>0$

Again, the proofs are easy.

Definition. When one of the assertions (a), (b), (a'), (b'), (A), (B), (C), (D), (E) of Lemmas 7 and 8 is true (id est, all of them), it will be said that the couple $(\mathbb{P}, \mathbb{D})$ is badly approximable.

We shall also need famous Minkowski's theorem on successive minima.

Lemma 9 (Minkowski's Successive Minima Theorem). For any convex set $E$ in $\mathbb{R}^{3}$ which is symmetric about $\mathbf{0}$, let $\Lambda_{i}(E)$, for $i=0,1$ or 2 , be the lower bound of the numbers $\lambda$ such that $\lambda E$ contains $(i+1)$ linearly independent integer vectors. Then, if the volume of $E$ is $8, \frac{1}{6} \leq \Lambda_{0}(E) \Lambda_{1}(E) \Lambda_{2}(E) \leq 1$ holds. See Cassels [5] (Cassels) Ch. VIII, page 201 and following, especially assertions $\{12\}$ and $\{13\}$ page 203, or [6] (Cassels), Theorem V page 156.

Proof of the b) and c) of Th. 2 (Dirichlet Properties). We suppose that the Hypothesis of the b) Property of Theorem 2 holds: there exists $c>0$ such 
that for any non-null integer point $\mathbf{h},\|\mathbf{h}\|^{2}\left\|\mathbf{h}^{\prime \prime}\right\|>c$. Then, by our Geometrical Transference Lemma, there exists $d>0$ such that for any non-null integer point $\mathbf{h},\left\|\mathbf{h}^{\prime}\right\|^{2}\left\|\mathbf{h}^{\prime \prime}\right\|>d$.

Like above, let $\Gamma_{R}$ be the cylinder: $\Gamma_{R}=\operatorname{Disk}^{\prime}(R)+\operatorname{Disk}^{\prime \prime}\left(\frac{4}{\pi R^{2}}\right)$.

Let's define $K_{0}=\left(\frac{\pi d}{4}\right)^{\frac{1}{3}}$. The inequality $\left\|\mathbf{h}^{\prime}\right\|^{2}\left\|\mathbf{h}^{\prime \prime}\right\|>d$ implies that there's no non-null integer point in $K_{0} \Gamma_{R}$. Then, for any $R, \Lambda_{0}\left(\Gamma_{R}\right) \geq$ $K_{0}$. In addition, we have $\Lambda_{0}\left(\Gamma_{R}\right) \Lambda_{1}\left(\Gamma_{R}\right) \Lambda_{2}\left(\Gamma_{R}\right) \leq 1$ and also $\Lambda_{1}\left(\Gamma_{R}\right) \geq$ $\Lambda_{0}\left(\Gamma_{R}\right) \geq K_{0}$, so that we get $K_{0}^{2} \Lambda_{2}\left(\Gamma_{R}\right) \leq 1$ and then $\Lambda_{2}\left(\Gamma_{R}\right)<K_{2}$, with $K_{2}=\frac{2}{K_{0}^{2}}$. Then, for each $R$, the cylinder $K_{2} \Gamma_{R}$ contains a free triplet of integer vectors $\left(\mathbf{h}_{0}, \mathbf{h}_{1}, \mathbf{h}_{2}\right)$.

Let now be $s$ in $S$, verifying $\frac{\left\|\mathbf{g}_{\mathrm{III}}^{\prime(s)}\right\|}{\rho^{(s)}}<L$. We may suppose $R=\frac{\rho^{(s)}}{K_{2}}$, so that

$$
K_{2} \Gamma_{R}=\operatorname{Disk}^{\prime}\left(\rho^{(s)}\right)+\operatorname{Disk}^{\prime \prime}\left(\frac{4 K_{2}^{3}}{\pi\left(\rho^{(s)}\right)^{2}}\right),
$$

which contains a free triplet of integer vectors $\left(\mathbf{h}_{0}, \mathbf{h}_{1}, \mathbf{h}_{2}\right)$, but the basis of which, Disk $\left(\rho^{(s)}\right)$, is contained in the "hexagon" $\mathbf{H}^{\prime(s)}$ generated by our algorithm. Then, by the Prism Lemma,

$\left\|\mathbf{g}_{\text {III }}^{\prime(s)}\right\|^{2}\left\|\mathbf{g}_{2}^{\prime \prime(s)}\right\| \leq L^{2}\left(\rho^{(s)}\right)^{2}\left\|\mathbf{g}_{2}^{\prime \prime(s)}\right\| \leq L^{2}\left(\rho^{(s)}\right)^{2}\left(\max _{i=0,1,2}\left\|\mathbf{h}_{i}^{\prime \prime(s)}\right\|\right) \leq \frac{4 K_{2}^{3} L^{2}}{\pi}$

and the main statement of $\mathbf{b}$ ) is proved. The second statement follows from this very result and from $\lim _{s \rightarrow+\infty, s \in S}\left\|\mathbf{g}_{\mathrm{III}}^{\prime(s)}\right\|=+\infty$ from Lemma 2 at the beginning of Section 2 .

Let's now verify the c) Property. We've just proved that under the Geometrical Theorem, for some $M, \sup _{s \in S}\left(\left\|\mathbf{g}_{\mathrm{III}}^{\prime(s)}\right\|^{2}\left\|\mathbf{g}_{2}^{\prime \prime}{ }_{2}^{(s)}\right\|\right) \leq M$ holds. Now, if $\varepsilon_{s}=\operatorname{det}\left(\mathbf{B}^{(s)}\right)=\operatorname{det}\left(\mathbf{G}^{(s)}\right)= \pm 1$, and if $(i, j, k)$ is a direct circular permutation of $(0,1,2)$, forgetting the indices, we have:

$$
\mathbf{b}_{i}=\varepsilon\left(\mathbf{g}_{j} \wedge \mathbf{g}_{k}\right) ; \mathbf{b}_{i}^{\prime}=\varepsilon\left(\mathbf{g}_{j}^{\prime \prime} \wedge \mathbf{g}_{k}^{\prime}+\mathbf{g}_{j}^{\prime} \wedge \mathbf{g}_{k}^{\prime \prime}\right) ; \mathbf{b}_{i}^{\prime \prime}=\varepsilon\left(\mathbf{g}_{j}^{\prime} \wedge \mathbf{g}_{k}^{\prime}\right) .
$$

Then for each $i,\left\|\mathbf{b}_{i}^{\prime}\right\| \leq 2\left\|\mathbf{g}_{\text {III }}^{\prime}\right\|\left\|\mathbf{g}_{2}^{\prime \prime}\right\|$, and $\left\|\mathbf{b}_{i}^{\prime \prime}\right\| \leq\left\|\mathbf{g}_{\text {III }}^{\prime}\right\|^{2}$. Then

$$
\left(\max _{i=0,1,2}\left\|\mathbf{b}_{i}^{\prime(s)}\right\|\right)^{2}\left(\max _{i=0,1,2}\left\|\mathbf{b}_{i}^{\prime \prime(s)}\right\|\right) \leq 4\left(\left\|\mathbf{g}_{\mathrm{III}}^{\prime(s)}\right\|^{2}\left\|\mathbf{g}_{2}^{\prime \prime(s)}\right\|\right)^{2} \leq 4 M^{2},
$$

and the main conclusion of the Part $\mathbf{c}$ ) is proved. Furthermore, we have seen that for each $s$ and each $i,\left\|\mathbf{b}_{i}^{\prime(s)}\right\| \leq 2\left\|\mathbf{g}_{\mathrm{III}}^{\prime(s)}\right\|\left\|\mathbf{g}_{2}^{\prime \prime(s)}\right\|$; in addition: 
$\left\|\mathbf{g}_{\mathrm{III}}^{\prime(s)}\right\|^{2}\left\|\mathbf{g}_{2}^{\prime \prime(s)}\right\| \leq M$ holds. Then, for each $s \in S,\left\|\mathbf{b}_{i}^{\prime(s)}\right\| \leq \frac{2 M}{\left\|\mathbf{g}_{\mathrm{III}}^{\prime(s)}\right\|}$. But, by the Lemma 2 of $\S 2.1, \lim _{s \rightarrow+\infty}\left\|\mathbf{g}_{\mathrm{III}}^{\prime(s)}\right\|=+\infty$. Then

$$
\lim _{s \rightarrow+\infty, s \in S}\left(\max _{i=0,1,2}\left\|\mathbf{b}_{i}^{(s)}\right\|\right)=0
$$

and the second part of $\mathbf{c}$ ) is proved.

\section{Demonstration of the Geometrical Theorem of $\S 1.3$.}

The demonstration of the Geometrical Theorem, Theorem 3. in Subsection 1.3., involves only very elementary geometry, but is a little long. In order to prove it, we first need some auxiliary sets and definitions.

\subsection{The area $A^{(s)}$ and the set $T$ (advances of $\left.\left\|\mathrm{g}_{\mathrm{III}}^{\prime(s)}\right\|\right)$.}

\section{Notation 2.}

* We'll denote by $A^{(s)}$ twice the area of the triangle $\left(\mathbf{g}_{0}^{\prime(s)} \mathbf{g}_{1}^{\prime(s)} \mathbf{g}_{2}^{\prime(s)}\right)$.

* We'll denote by $\left(\mathbf{g}_{\mathrm{I}}^{\prime(s)}, \mathbf{g}_{\mathrm{II}}^{\prime(s)}, \mathbf{g}_{\mathrm{III}}^{\prime(s)}\right)$ the permutation of $\left(\mathbf{g}_{0}^{\prime(s)}, \mathbf{g}_{1}^{\prime(s)}, \mathbf{g}_{2}^{\prime(s)}\right)$ such that $\left\|\mathbf{g}_{\mathrm{I}}^{\prime(s)}\right\| \leq\left\|\mathbf{g}_{\mathrm{II}}^{\prime(s)}\right\| \leq\left\|\mathbf{g}_{\mathrm{III}}^{\prime(s)}\right\|$.

* In the Smallest Vector Algorithm, let's denote by $T$ the set of all integers s such that $\left\|\mathbf{g}_{\text {III }}^{\prime(s)}\right\|<\left\|\mathbf{g}_{\mathrm{III}}^{(s+1)}\right\|$.

* The set $T$ is infinite, because by Lemma 2, we have:

$$
\lim _{s \rightarrow+\infty}\left\|\mathbf{g}_{\mathrm{III}}^{\prime(s)}\right\|=+\infty \text {. }
$$

Remark. a) $A^{(s)}=\left\|\mathbf{g}_{\mathrm{I}}^{\prime(s)} \wedge \mathbf{g}_{\mathrm{II}}^{\prime(s)}+\mathbf{g}_{\mathrm{II}}^{\prime(s)} \wedge \mathbf{g}_{\mathrm{III}}^{\prime(s)}+\mathbf{g}_{\mathrm{III}}^{\prime(s)} \wedge \mathbf{g}_{\mathrm{I}}^{\prime(s)}\right\| ;$

b) It's easy to establish that for some $i$ and $j$ among $\{0,1,2\}$, we have $\left.A^{(s+1)}=A^{(s)}+\left\|\mathbf{g}_{i}^{(s)} \wedge \mathbf{g}_{j}^{(s)}\right\| ; \mathbf{c}\right)$ Then the sequence $\left(A^{(s)}\right)_{s \in \mathbb{N}}$ is increasing.

Notation 3. In this paper, for two non null vectors $\mathbf{a}$ and $\mathbf{b}$ in $\mathbb{R}^{3}$, we shall consider the angle of these two vectors corresponding to the canonical euclidean norm, and the measure of this angle which belongs to ] $\pi ; \pi]$. This measure will be denoted either by " $\measuredangle(\mathbf{a}, \mathbf{b}) "$ ", or, more simply, when no ambiguity can occur, by "(a, b)".

Lemma 10 (Geometry on T). In the Smallest Vector Algorithm, for any $s \in T,\left\|\mathbf{g}_{\mathrm{II}}^{\prime(s)}\right\|>\frac{1}{2}\left\|\mathbf{g}_{\mathrm{III}}^{\prime(s)}.\right\|$. Moreover, for $i, j \in\{0,1,2\}$ we have:

$$
\frac{\pi}{3}<\left|\measuredangle\left(\mathbf{g}_{i}^{\prime(s)}, \mathbf{g}_{j}^{\prime(s)}\right)\right|
$$


Proof. Let $i, j \in\{0,1,2\}, i \neq j$. We have $\left\|\mathbf{g}_{\mathrm{III}}^{(s+1)}\right\| \leq\left\|\mathbf{g}_{i}^{\prime(s)}-\mathbf{g}_{j}^{\prime(s)}\right\|$ and then $\left\|\mathbf{g}_{\text {III }}^{\prime(s+1)}\right\|^{2} \leq\left\|\mathbf{g}_{i}^{\prime(s)}\right\|^{2}+\left\|\mathbf{g}_{j}^{\prime(s)}\right\|^{2}\left(1-2 \cos \left(\mid\left(\mathbf{g}_{i}^{\prime(s)}, \mathbf{g}_{j}^{\prime(s)}\right) \|\right) \frac{\left\|\mathbf{g}_{i}^{\prime(s)}\right\|}{\left\|\mathbf{g}_{j}^{\prime(s)}\right\|}\right)$.

Without loss of generality, we may suppose $\left\|\mathbf{g}_{i}^{\prime(s)}\right\| \geq\left\|\mathbf{g}_{j}^{\prime(s)}\right\|$. Then, by the cos formula above, if $\left|\measuredangle\left(\mathbf{g}_{i}^{\prime(s)}, \mathbf{g}_{j}^{\prime(s)}\right)\right| \leq \frac{\pi}{3}$ would hold, we would have

$$
\left\|\mathbf{g}_{\text {III }}^{\prime(s+1)}\right\|^{2} \leq\left\|\mathbf{g}_{i}^{\prime(s)}\right\|^{2} \leq\left\|\mathbf{g}_{\text {III }}^{\prime(s)}\right\|^{2}
$$

and $s$ wouldn't be in $T$. Then $\left|\measuredangle\left(\mathbf{g}_{i}^{\prime(s)}, \mathbf{g}_{j}^{\prime(s)}\right)\right|>\frac{\pi}{3}$ holds. Moreover

$$
\left\|\mathbf{g}_{\text {III }}^{\prime(s)}\right\|<\left\|\mathbf{g}_{\text {III }}^{\prime(s+1)}\right\| \leq\left\|\mathbf{g}_{\text {I }}^{\prime(s)}-\mathbf{g}_{\text {II }}^{\prime(s)}\right\| \leq 2\left\|\mathbf{g}_{\text {II }}^{\prime(s)}\right\|
$$

Hence $\left\|\mathbf{g}_{\text {II }}^{\prime(s)}\right\|>\frac{1}{2}\left\|\mathbf{g}_{\text {III }}^{\prime(s)}\right\|$.

4.2. The notion of "Needling". As A.J. Brentjes has already noted in [3], if we want our vectors to have good approximation qualities, their projections $\mathbf{g}_{0}^{\prime}, \mathbf{g}_{1}^{\prime}, \mathbf{g}_{2}^{\prime}$ on $\mathbb{P}$ must avoid the needling, i.e. flattening phenomenon.

We're going to define and study this phenomenon, but first we need the following Lemma in elementary geometry. It is very easy and its proof will be omitted here.

Lemma 11. Let $\mathbf{a}$ and $\mathbf{b}$ be two vectors of the plane, with $0<\|\mathbf{b}\| \leq\|\mathbf{a}\|$. Let's suppose that there exist real numbers $\varepsilon>0$ and $M>0$ such that:

$$
\varepsilon \leq|\measuredangle(\mathbf{a}, \mathbf{b})| \leq \pi-\varepsilon \text { and } \frac{\|\mathbf{b}\|}{\|\mathbf{a}\|} \geq M
$$

Let $\rho$ be the radius of the greatest disk centered at $\mathbf{0}$ and included in the parallelogram $(\mathbf{a}, \mathbf{b},(-\mathbf{a}),(-\mathbf{b}))$. Then there exists a real number $M^{\prime}>0$, depending only on $\varepsilon$ and $M$, such that $\frac{\rho}{\|\mathbf{a}\|} \geq M^{\prime}$.

As an immediate corollary, we have:

Lemma 12. (Needling Sequence of Parallelograms) Let $\left(\mathbf{a}^{(s)}, \mathbf{b}^{(s)},\left(-\mathbf{a}^{(s)}\right),\left(-\mathbf{b}^{(s)}\right)\right)$ be a sequence of parallelograms, with:

$$
0<\left\|\mathbf{b}^{(s)}\right\| \leq\left\|\mathbf{a}^{(s)}\right\|
$$

If these parallelograms are needling, id est if $\lim _{s \rightarrow+\infty} \frac{\rho^{(s)}}{\left\|\mathbf{a}^{(s)}\right\|}=0$, where $\rho$ is defined like in the preceding Lemma, then

$$
\lim _{s \rightarrow+\infty} \sin \left(\mathbf{a}^{(s)}, \mathbf{b}^{(s)}\right) \times \frac{\left\|\mathbf{b}^{(s)}\right\|}{\left\|\mathbf{a}^{(s)}\right\|}=0
$$


Proof. If the conclusion were false, then, for some $\eta>0$ and for any $s$ in some infinite set $U$, we should have: $\sin \left(\mathbf{a}^{(s)}, \mathbf{b}^{(s)}\right) \geq \eta$ and $\frac{\left\|\mathbf{b}^{(s)}\right\|}{\left\|\mathbf{a}^{(s)}\right\|} \geq \eta$, and then, by the preceding Lemma, $\frac{\rho^{(s)}}{\left\|\mathbf{a}^{(s)}\right\|} \geq M^{\prime}$ for some $M^{\prime}$ and for $s \in U$. But this negates the hypothesis of our Lemma. Then the conclusion is true.

This last Lemma leads to the more important Lemma, which describes the needling phenomenon on the set $T$.

Lemma 13. (Needling Triangles) The three following assumptions are logically equivalent: $\boldsymbol{a}) \lim _{s \rightarrow+\infty, s \in T} \frac{A^{(s)}}{\left\|\mathbf{g}_{\mathrm{II}}^{(s)}\right\|^{2}}=0$;

b) $\lim _{s \rightarrow+\infty, s \in T} \frac{\rho^{(s)}}{\left\|\mathbf{g}_{\text {III }}^{(s)}\right\|}=0$, where $\rho^{(s)}$ is the radius of the greatest disk centered at $\mathbf{0}$ and included in the convex hull:

$\mathbf{H}^{\prime(s)}=\operatorname{CONV}\left(\mathbf{g}_{0}^{\prime}, \mathbf{g}_{1}^{\prime}, \mathbf{g}_{2}^{\prime},-\mathbf{g}_{0}^{\prime},-\mathbf{g}_{1}^{\prime},-\mathbf{g}_{2}^{\prime}\right)$ in $\mathbb{P} ;$

c ) $\lim _{s \rightarrow+\infty, s \in T}\left(\frac{\left\|\mathbf{g}_{\text {I }}^{\prime(s)}\right\|}{\left\|\mathbf{g}_{\text {II }}^{\prime(s)}\right\|}+\left(\pi-\left|\measuredangle\left(\mathbf{g}_{\text {II }}^{\prime(s)}, \mathbf{g}_{\text {III }}^{(s)}\right)\right|\right)\right)=0$.

Proof. First, let's prove a) $\Rightarrow$ b). Omitting the indices ${ }^{(s)}$, we have:

$$
\mathbf{H}^{\prime}=\operatorname{CONV}\left(\mathbf{g}_{0}^{\prime}, \mathbf{g}_{1}^{\prime}, \mathbf{g}_{2}^{\prime},-\mathbf{g}_{0}^{\prime},-\mathbf{g}_{1}^{\prime}-, \mathbf{g}_{2}^{\prime}\right) \text { in } \mathbb{P} .
$$

Let now $\mathbf{K}^{\prime}$ be the convex hull of all the points $2 \mathbf{g}_{i}^{\prime}-\mathbf{g}_{j}^{\prime}$, with $i \neq j$ and $\{i, j\} \subset\{0,1,2\}$. Each $\mathbf{g}_{i}^{\prime}$ belongs to $\mathbf{K}^{\prime}$, because $\mathbf{g}_{i}^{\prime}=\frac{2}{3}\left(2 \mathbf{g}_{i}^{\prime}-\mathbf{g}_{j}^{\prime}\right)+$ $\frac{1}{3}\left(2 \mathbf{g}_{j}^{\prime}-\mathbf{g}_{i}^{\prime}\right)$. In addition, the projection of the cofactors relation on $\mathbb{P}$ leads to:

$$
\|\mathbf{X}\|\left\|\mathbf{b}_{0}^{\prime \prime}\right\| \cdot \mathbf{g}_{0}^{\prime}+\|\mathbf{X}\|\left\|\mathbf{b}_{1}^{\prime \prime}\right\| \cdot \mathbf{g}_{1}^{\prime}+\|\mathbf{X}\|\left\|\mathbf{b}_{2}^{\prime \prime}\right\| \cdot \mathbf{g}_{2}^{\prime}=\mathbf{0} .
$$

Then, $\mathbf{0}$ is in the triangle $\mathbf{g}_{0}^{\prime} \mathbf{g}_{1}^{\prime} \mathbf{g}_{2}^{\prime}$. Let $F_{0}$ be the homothety with center $\mathbf{g}_{0}^{\prime}$ and with scaling 2 . Then $F_{0}(\mathbf{0})=-\mathbf{g}_{0}^{\prime}$ is inside $F\left(\mathbf{g}_{0}^{\prime} \mathbf{g}_{1}^{\prime} \mathbf{g}_{2}^{\prime}\right)$, which is the triangle with summits $\mathbf{g}_{2}^{\prime} ; 2 \mathbf{g}_{1}^{\prime}-\mathbf{g}_{0}^{\prime} ; 2 \mathbf{g}_{2}^{\prime}-\mathbf{g}_{0}^{\prime}$. Then $\left(-\mathbf{g}_{0}^{\prime}\right)$, and, in the same way, $\left(-\mathbf{g}_{1}^{\prime}\right)$ and $\left(-\mathbf{g}_{2}^{\prime}\right)$, belong to $\mathbf{K}^{\prime}$. Then $\mathbf{H}^{\prime} \subset \mathbf{K}^{\prime}$; then $\pi\left(\rho^{(s)}\right)^{2} \leq$ area $\left(\mathbf{K}^{\prime(s)}\right)$. But $\mathbf{K}^{\prime(s)}$ is formed with 13 triangles, each of them isometric to the triangle $\mathbf{g}_{0}^{\prime} \mathbf{g}_{1}^{\prime} \mathbf{g}_{2}^{\prime}$. Then:

$$
\pi\left(\rho^{(s)}\right)^{2} \leq \operatorname{area}\left(\mathbf{K}^{\prime(s)}\right) \leq 13 A^{(s)} .
$$

Then, using a), we have $\pi\left(\rho^{(s)}\right)^{2} \leq\left\|\mathbf{g}_{\mathrm{III}}^{(s)}\right\|^{2} \varepsilon(s)$, with $\lim _{x \rightarrow+\infty, x \in T} \varepsilon(s)=0$. This implies b). 
Second, let's prove $\mathrm{b}) \Rightarrow \mathrm{c}$ ). If $\mathrm{b})$ is true, we also have $\lim _{s \rightarrow+\infty, s \in T} \frac{\rho^{(s)}}{\left\|\mathbf{g}_{\mathrm{III}}^{\prime(s)}\right\|}=$ 0 , if $\rho^{(s)}$ is the radius of the greatest disk centered at $\mathbf{0}$ and included in the parallelogram with summits $\mathbf{g}_{\mathrm{II}}^{\prime}, \mathbf{g}_{\mathrm{III}}^{\prime},-\mathbf{g}_{\mathrm{II}}^{\prime},-\mathbf{g}_{\mathrm{III}}^{\prime}$. Then, by the last Lemma:

$$
\lim _{s \rightarrow+\infty, s \in T} \sin \left(\mathbf{g}_{\mathrm{II}}^{\prime(s)}, \mathbf{g}_{\mathrm{III}}^{(s)}\right) \times \frac{\left\|\mathbf{g}_{\mathrm{II}}^{(s)}\right\|}{\left\|\mathbf{g}_{\mathrm{III}}^{\prime(s)}\right\|}=0
$$

But, by the Lemma "Geometry on $T$ " of the last subsection above, $\frac{\| \mathbf{g}_{\text {II }}^{(s)}}{\left\|\mathbf{g}_{\text {III }}^{\prime(s)}\right\|} \geq$ $\frac{1}{2}$ holds. Then $\lim _{s \rightarrow+\infty, s \in T} \sin \left(\mathbf{g}_{\mathrm{II}}^{\prime(s)}, \mathbf{g}_{\mathrm{III}}^{\prime(s)}\right)=0$. By the same Lemma:

$$
\left|\measuredangle\left(\mathbf{g}_{\mathrm{II}}^{\prime(s)}, \mathbf{g}_{\mathrm{III}}^{\prime(s)}\right)\right|>\frac{\pi}{3}
$$

Then: $\lim _{s \rightarrow+\infty, s \in T}\left(\pi-\left|\measuredangle\left(\mathbf{g}_{\mathrm{II}}^{(s)}, \mathbf{g}_{\mathrm{III}}^{\prime(s)}\right)\right|\right)=0$.

This last result, with the help of $\left|\measuredangle\left(\mathbf{g}_{\mathrm{I}}^{\prime(s)}, \mathbf{g}_{\mathrm{III}}^{\prime(s)}\right)\right|>\frac{\pi}{3}$, by the same Lemma, leads to: $\limsup _{s \rightarrow+\infty, s \in T}\left|\measuredangle\left(\mathbf{g}_{\mathrm{I}}^{\prime(s)}, \mathbf{g}_{\mathrm{II}}^{\prime(s)}\right)\right|<\frac{2 \pi}{3}$. We also have by the same Lemma, $\left|\measuredangle\left(\mathbf{g}_{\mathrm{I}}^{\prime(s)}, \mathbf{g}_{\mathrm{II}}^{\prime(s)}\right)\right|>\frac{\pi}{3}$. Then: $\liminf _{s \rightarrow+\infty, s \in T}\left|\sin \left(\mathbf{g}_{\mathrm{I}}^{\prime(s)}, \mathbf{g}_{\mathrm{II}}^{(s)}\right)\right|>0$.

But, if b) is true, $\lim _{s \rightarrow+\infty, s \in T} \frac{\rho^{(s)}}{\left\|\mathbf{g}_{\text {III }}^{\prime(s)}\right\|}=0$ holds, if $\rho^{(s)}$ is the radius of the greatest disk centered at $\mathbf{0}$ and included in the other parallelogram, with summits $\mathbf{g}_{\mathrm{I}}^{\prime}, \mathbf{g}_{\mathrm{III}}^{\prime},-\mathbf{g}_{\mathrm{I}}^{\prime},-\mathbf{g}_{\mathrm{III}}^{\prime}$, and then, by $\frac{\left\|\mathbf{g}_{\mathrm{II}}^{(s)}\right\|}{\left\|\mathbf{g}_{\mathrm{III}}^{(s)}\right\|} \geq \frac{1}{2}$, we also have: $\lim _{s \rightarrow+\infty, s \in T} \frac{\rho^{(s)}}{\left\|\mathbf{g}_{I I}^{\prime(s)}\right\|}=0$. Then, by the last Lemma on the needling parallelograms: $\lim _{s \rightarrow+\infty, s \in T} \sin \left(\mathbf{g}_{\mathrm{I}}^{\prime(s)}, \mathbf{g}_{\mathrm{II}}^{(s)}\right) \times \frac{\left\|\mathbf{g}_{\mathrm{I}}^{\prime(s)}\right\|}{\left\|\mathbf{g}_{\mathrm{II}}^{\prime(s)}\right\|}=0$, and using the lim inf above, we obtain: $\lim _{s \rightarrow+\infty, s \in T}\left\|\frac{\mathbf{g}_{\mathrm{I}}^{(s)}}{\left\|\mathbf{g}_{\mathrm{II}}^{((s)}\right\|}\right\|=0$. The proof of the part $\left.\mathrm{b}\right) \Rightarrow \mathrm{c}$ ) is done.

Finally, the implication $c) \Rightarrow a$ ) is obvious.

In order to refute the needling phenomenon, we're going to study what happens when the projections on $\mathbb{P}$ are "almost flat". This will allow us to prove that the needling CANNOT happen. 


\subsection{Almost Flat Triangles. Set $T^{*}$ of indices.}

Notation 4. $T^{*}$ will denote the set of all integers $s \in T$ such that the triangle $\mathbf{g}_{0}^{\prime(s)} \mathbf{g}_{1}^{\prime(s)} \mathbf{g}_{2}^{\prime(s)}$ is "almost flat", namely such that: $\| \frac{\mathbf{g}_{\mathrm{I}}^{\prime(s)} \|}{\left\|\mathbf{g}_{\mathrm{II}}^{\prime(s)}\right\|} \leq 0.1$ and $\left|\measuredangle\left(\mathbf{g}_{\text {III }}^{\prime(s)}, \mathbf{g}_{\text {II }}^{\prime(s)}\right)\right| \geq \frac{30 \pi}{31}$.

Lemma 14 (Flat Triangle Lemma). If $s \in T^{*}$ (i.e. if the triangle $\mathbf{g}_{0}^{\prime(s)} \mathbf{g}_{1}^{\mathbf{\prime}^{(s)}}$ $\mathbf{g}_{2}^{(s)}$ is "almost flat"), then we also have the following relations:

$$
\frac{15 \pi}{31} \leq\left|\measuredangle\left(\mathbf{g}_{\text {III }}^{\prime(s)}, \mathbf{g}_{\mathrm{I}}^{\prime(s)}\right)\right| \leq \frac{17 \pi}{31} \text { and } \frac{15 \pi}{31} \leq\left|\measuredangle\left(\mathbf{g}_{\text {II }}^{\prime(s)}, \mathbf{g}_{\mathrm{I}}^{\prime(s)}\right)\right| \leq \frac{17 \pi}{31},
$$

and also:

$$
\frac{\left\|\mathbf{g}_{\text {II }}^{\prime(s)}\right\|}{\left\|\mathbf{g}_{\text {III }}^{\prime(s)}\right\|} \geq 0.979,\left\|\mathbf{g}_{\text {II }}^{(s)}+\mathbf{g}_{\mathrm{III}}^{\prime(s)}\right\| \leq 0.23\left\|\mathbf{g}_{\mathrm{III}}^{(s)}\right\|
$$

and:

$$
\left\|\mathbf{g}_{\mathrm{I}}^{\prime(s)}-\mathbf{g}_{\text {II }}^{\prime(s)}-\mathbf{g}_{\text {III }}^{\prime(s)}\right\| \leq 0.33\left\|\mathbf{g}_{\text {III }}^{\prime(s)}\right\|
$$

Proof. Because in $T,\left\|\mathbf{g}_{i}^{\prime(s)}-\mathbf{g}_{j}^{\prime(s)}\right\| \geq\left\|\mathbf{g}_{\mathrm{III}}^{(s+1)}\right\| \geq\left\|\mathbf{g}_{\mathrm{III}}^{(s)}\right\|$ holds, both following inequalities also hold:

$$
\left\|\mathbf{g}_{\text {III }}^{\prime(s)}-\mathbf{g}_{\mathrm{I}}^{\prime(s)}\right\| \geq\left\|\mathbf{g}_{\text {III }}^{\prime(s)}\right\| \text { and }\left\|\mathbf{g}_{\text {II }}^{\prime(s)}-\mathbf{g}_{\mathrm{I}}^{\prime(s)}\right\| \geq\left\|\mathbf{g}_{\text {III }}^{\prime(s)}\right\| \geq\left\|\mathbf{g}_{\text {II }}^{\prime(s)}\right\| .
$$

The first inequality leads to:

$$
\left\|\mathbf{g}_{\text {III }}^{\prime(s)}\right\|^{2} \leq\left\|\mathbf{g}_{\text {III }}^{\prime(s)}\right\|^{2}+\left\|\mathbf{g}_{\text {I }}^{\prime(s)}\right\|\left\|\mathbf{g}_{\text {III }}^{\prime(s)}\right\|\left(\frac{\left\|\mathbf{g}_{\text {I }}^{\prime(s)}\right\|}{\left\|\mathbf{g}_{\text {III }}^{\prime(s)}\right\|}-2 \cos \left(\mathbf{g}_{\text {III }}^{\prime(s)}, \mathbf{g}_{\text {I }}^{(s)}\right)\right)
$$

Then: $0.1-2 \cos \left(\mathbf{g}_{\mathrm{III}}^{\prime(s)}, \mathbf{g}_{\mathrm{I}}^{\prime(s)}\right) \geq 0$; then: $\cos \left(\mathbf{g}_{\mathrm{III}}^{\prime(s)}, \mathbf{g}_{\mathrm{I}}^{\prime(s)}\right) \leq 0.05$. Hence: $\left|\measuredangle\left(\mathbf{g}_{\text {III }}^{\prime(s)}, \mathbf{g}_{\mathrm{I}}^{\prime(s)}\right)\right| \geq \frac{15 \pi}{31}$. Hence $\left|\measuredangle\left(\mathbf{g}_{\text {II }}^{\prime(s)}, \mathbf{g}_{\mathrm{I}}^{(s)}\right)\right| \leq \frac{17 \pi}{31}$.

In the same way, we obtain:

$$
\left|\measuredangle\left(\mathbf{g}_{I I}^{\prime(s)}, \mathbf{g}_{\mathrm{I}}^{\prime(s)}\right)\right| \geq \frac{15 \pi}{31} \text { and }\left|\measuredangle\left(\mathbf{g}_{\mathrm{III}}^{\prime(s)}, \mathbf{g}_{\mathrm{I}}^{\prime(s)}\right)\right| \leq \frac{17 \pi}{31} .
$$

Again, let's use the inequality: $\left\|\mathbf{g}_{\mathrm{III}}^{\prime(s)}\right\|^{2} \leq\left\|\mathbf{g}_{\mathrm{II}}^{\prime(s)}-\mathbf{g}_{\mathrm{I}}^{\prime(s)}\right\|^{2}$. Then

$$
\left\|\mathbf{g}_{\text {III }}^{\prime(s)}\right\|^{2} \leq\left\|\mathbf{g}_{\text {II }}^{\prime(s)}\right\|^{2}+\left\|\mathbf{g}_{\mathrm{I}}^{\prime(s)}\right\|^{2}-2 \cos \left(\mathbf{g}_{\mathrm{II}}^{\prime(s)}, \mathbf{g}_{\mathrm{I}}^{\prime(s)}\right)\left\|\mathbf{g}_{\mathrm{II}}^{\prime(s)}\right\|\left\|\mathbf{g}_{\mathrm{I}}^{(s)}\right\| .
$$


But $-2 \cos \left(\left(\mathbf{g}_{\mathrm{II}}^{\prime(s)}, \mathbf{g}_{\mathrm{I}}^{\prime(s)}\right)\right) \leq-2 \cos \left(\frac{17 \pi}{31}\right) \leq 0.31$. Then, dividing by $\left\|\mathbf{g}_{\text {III }}^{\prime(s)}\right\|^{2}$, we obtain

$$
1 \leq \frac{\left\|\mathbf{g}_{\mathrm{II}}^{\prime(s)}\right\|^{2}}{\left\|\mathbf{g}_{\mathrm{III}}^{\prime(s)}\right\|^{2}}+0.01+0.031 \frac{\left\|\mathbf{g}_{\mathrm{II}}^{\prime(s)}\right\|}{\left\|\mathbf{g}_{\mathrm{III}}^{\prime(s)}\right\|} \text {, i.e.: } x^{2}+0.031 x-0.99 \geq 0
$$

with $x=\frac{\| \mathbf{g}_{\mathrm{II}}^{(s)}}{\| \mathbf{g}_{\mathrm{III}}^{\prime(s)}} \|$. This implies $x=\left\|\frac{\mathbf{g}_{\mathrm{II}}^{\prime(s)}}{\| \mathbf{g}_{\mathrm{III}}^{\prime(s)}}\right\| \geq 0.979$. We have

$$
\left\|\mathbf{g}_{\text {II }}^{\prime(s)}+\mathbf{g}_{\text {III }}^{(s)}\right\|^{2} \leq\left\|\mathbf{g}_{\text {II }}^{\prime(s)}\right\|^{2}+\left\|\mathbf{g}_{\text {III }}^{\prime(s)}\right\|^{2}+2 \cos \left(\frac{30 \pi}{31}\right)\left\|\mathbf{g}_{\text {III }}^{\prime(s)}\right\|\left\|\mathbf{g}_{\text {II }}^{\prime(s)}\right\|,
$$

then

$$
\left\|\mathbf{g}_{\text {II }}^{\prime(s)}+\mathbf{g}_{\text {III }}^{\prime(s)}\right\|^{2} \leq 2\left\|\mathbf{g}_{\text {III }}^{\prime(s)}\right\|^{2}-1.9897 \times 0.979\left\|\mathbf{g}_{\text {III }}^{\prime(s)}\right\|^{2}
$$

then

$$
\left\|\mathbf{g}_{\text {II }}^{\prime(s)}+\mathbf{g}_{\text {III }}^{\prime(s)}\right\| \leq \sqrt{0.0521}\left\|\mathbf{g}_{\text {III }}^{\prime(s)}\right\| \leq 0.23\left\|\mathbf{g}_{\text {III }}^{\prime(s)}\right\|
$$

Hence the last conclusion is obtained.

\subsection{From a Lemma to the Geometrical Theorem (Theorem 3).}

Notation 5. The sequence $\left(\alpha^{(s)}\right)$ is defined by: $\alpha^{(s)}=\frac{A^{(s)}}{\left\|\mathbf{g}_{\text {III }}^{\prime(s)}\right\|^{2}}$.

If $\limsup _{s \rightarrow+\infty} \alpha^{(s)}>0$, then the algorithm is balanced, i.e. the triangles on $\mathbb{P}$ do not needle.

Lemma 15. (Monotonic Subsequence Lemma) Let $[m ;+\infty[$ be an interval of $\mathbb{N}$ such that $\left[m ;+\infty\left[\cap T \subset T^{*}\right.\right.$, which means that every advancing triangle with its range in $\left[m ;+\infty\left[\right.\right.$ is almost flat. Then the sequence $\left(\alpha^{(s)}\right)$ is increasing on $[m ;+\infty[\cap T$, which means that for any $s, t \in[m ;+\infty[\cap T$ with $s \leq t, \frac{A^{(s)}}{\left\|\mathbf{g}_{\mathrm{III}}^{(s)}\right\|^{2}} \leq \frac{A^{(t)}}{\left\|\mathbf{g}_{\mathrm{III}}^{\prime(t)}\right\|^{2}}$ holds.

Let's admit this Lemma, which is proved in the next Subsection. Then we can demonstrate the Geometrical Theorem, Theorem 3.

Proof of Theorem 3. Let's suppose that the conclusion of the geometrical Lemma is FALSE, namely that $\lim _{s \rightarrow+\infty, s \in T} \frac{\rho^{(s)}}{\left\|\mathbf{g}_{\mathrm{III}}^{(s)}\right\|}=0$. Then, by the Lemma on needling triangles of the second subsection above, $\lim _{s \rightarrow+\infty, s \in T} \frac{A^{(s)}}{\left\|\mathbf{g}_{\mathrm{III}}^{\prime(s)}\right\|^{2}}=0$. 
By the same Lemma, we have

$$
\lim _{s \rightarrow+\infty, s \in T}\left(\frac{\left\|\mathbf{g}_{\mathrm{I}}^{(s)}\right\|}{\left\|\mathbf{g}_{\mathrm{II}}^{(s)}\right\|}+\left(\pi-\mid \measuredangle\left(\mathbf{g}_{\mathrm{II}}^{\prime(s)}, \mathbf{g}_{\mathrm{III}}^{\prime(s)}\right)\right)\right)=0
$$

Then, there exists an integer $m$ such that $\left[m ;+\infty\left[\cap T \subset T^{*}\right.\right.$, which means that with a range great enough, any advancing triangle is almost flat. Then, by the Monotonic Subsequence Lemma, the sequence of the $\alpha^{(s)}=\frac{A^{(s)}}{\left\|\mathbf{g}_{\text {III }}^{\prime(s)}\right\|^{2}}$ with $s \geq m$ is increasing on $T$, which is infinite. This is contradictory with $\lim _{s \rightarrow+\infty, s \in T} \frac{A^{(s)}}{\left\|\mathbf{g}_{\text {III }}^{\prime(s)}\right\|^{2}}=0$. Then, we have $\limsup _{s \rightarrow+\infty, s \in T} \frac{\rho^{(s)}}{\left\|\mathbf{g}_{\text {III }}^{\prime(s)}\right\|}>0$ and the

\section{Geometrical Theorem is proved.}

This Monotonic Subsequence Lemma has now to be proved.

4.5. Proof of the Monotonic Subsequence Lemma. Let $s$ be an integer in the interval $\left[m ;+\infty\left[\cap T \subset T^{*}\right.\right.$ as in the Hypothesis. Let's denote $s^{\prime}$ the successor of $s$ in $T$, i.e. the smallest integer $t$ in $T$ such that $s<t$

In order to establish our Lemma, it suffices to show that

$$
\frac{A^{(s)}}{\left\|\mathbf{g}_{\text {III }}^{\prime(s)}\right\|^{2}} \leq \frac{A^{\left(s^{\prime}\right)}}{\left\|\mathbf{g}_{\text {III }}^{\prime\left(s^{\prime}\right)}\right\|^{2}}
$$

We have four cases: Either $\mathbf{g}_{\mathrm{III}}^{(s+1)}=\mathbf{g}_{\mathrm{I}}^{(s)}-\mathbf{g}_{\mathrm{II}}^{(s)}$ (Case $(\mathrm{I} \backslash \mathrm{II})$ ), or $\mathbf{g}_{\mathrm{III}}^{\prime(s+1)}=\mathbf{g}_{\mathrm{I}}^{\prime(s)}-\mathbf{g}_{\mathrm{III}}^{(s)}(\mathrm{I} \backslash \mathrm{III})$, or $\mathbf{g}_{\mathrm{III}}^{\prime(s+1)}=\mathbf{g}_{\mathrm{II}}^{\prime(s)}-\mathbf{g}_{\mathrm{I}}^{\prime(s)}(\mathrm{II} \backslash \mathrm{I})$, or $\mathbf{g}_{\text {III }}^{\prime(s+1)}=\mathbf{g}_{\text {III }}^{\prime(s)}-\mathbf{g}_{\mathrm{I}}^{\prime(s)}($ III $\backslash \mathrm{I})$.

We're going to prove the inequality (Ineq 4.1) only in the case (II $\backslash \mathrm{I}$ ). The demonstration is similar, or easier, in the three other cases.

Case $(\mathrm{II} \backslash \mathrm{I}): \mathbf{g}_{\mathrm{III}}^{\prime(s+1)}=\mathbf{g}_{\mathrm{II}}^{(s)}-\mathbf{g}_{\mathrm{I}}^{\prime(s)}$.

We have to obtain first

$$
\frac{A^{(s)}}{\left\|\mathbf{g}_{\mathrm{III}}^{(s)}\right\|^{2}} \leq \frac{A^{(s+1)}}{\left\|\mathbf{g}_{\mathrm{III}}^{(s+1)}\right\|^{2}} \text {. i.e. } \frac{A^{(s+1)}}{A^{(s)}} \geq \frac{\left\|\mathbf{g}_{\mathrm{II}}^{\prime(s)}-\mathbf{g}_{\mathrm{I}}^{\prime(s)}\right\|^{2}}{\left\|\mathbf{g}_{\mathrm{III}}^{\prime(s)}\right\|^{2}}
$$

It's sufficient to show:

$$
\frac{A^{(s+1)}}{A^{(s)}} \geq\left(1+\frac{\left\|\mathbf{g}_{\mathrm{I}}^{(s)}\right\|}{\left\|\mathbf{g}_{\mathrm{III}}^{\prime(s)}\right\|}\right)^{2}
$$


But:

$$
\begin{aligned}
\frac{A^{(s+1)}}{A^{(s)}} & =\frac{A^{(s)}+\left\|\mathbf{g}_{\mathrm{III}}^{\prime(s)} \wedge \mathbf{g}_{\mathrm{I}}^{\prime(s)}\right\|}{A^{(s)}} \\
& =1+\frac{\left\|\mathbf{g}_{\mathrm{III}}^{\prime(s)} \wedge \mathbf{g}_{\mathrm{I}}^{\prime(s)}\right\|}{\left\|\mathbf{g}_{\mathrm{I}}^{\prime(s)} \wedge \mathbf{g}_{\mathrm{II}}^{\prime(s)}+\mathbf{g}_{\mathrm{II}}^{\prime(s)} \wedge \mathbf{g}_{\mathrm{III}}^{\prime(s)}+\mathbf{g}_{\mathrm{III}}^{\prime(s)} \wedge \mathbf{g}_{\mathrm{I}}^{\prime(s)}\right\|}
\end{aligned}
$$

It's sufficient to show:

$$
1+\frac{\left\|\mathbf{g}_{\mathrm{III}}^{\prime(s)} \wedge \mathbf{g}_{\mathrm{I}}^{\prime(s)}\right\|}{\left\|\mathbf{g}_{\mathrm{I}}^{\prime(s)} \wedge \mathbf{g}_{\mathrm{II}}^{\prime(s)}+\mathbf{g}_{\mathrm{II}}^{\prime(s)} \wedge \mathbf{g}_{\mathrm{III}}^{\prime(s)}+\mathbf{g}_{\mathrm{III}}^{(s)} \wedge \mathbf{g}_{\mathrm{I}}^{\prime(s)}\right\|} \geq\left(1+\frac{\left\|\mathbf{g}_{\mathrm{I}}^{\prime(s)}\right\|}{\left\|\mathbf{g}_{\mathrm{III}}^{\prime(s)}\right\|}\right)^{2}
$$

i.e.:

$$
\frac{\left\|\mathbf{g}_{\mathrm{III}}^{\prime(s)} \wedge \mathbf{g}_{\mathrm{I}}^{\prime(s)}\right\|}{\left\|\mathbf{g}_{\mathrm{I}}^{\prime(s)} \wedge \mathbf{g}_{\mathrm{II}}^{\prime(s)}+\mathbf{g}_{\mathrm{II}}^{\prime(s)} \wedge \mathbf{g}_{\mathrm{III}}^{\prime(s)}+\mathbf{g}_{\mathrm{III}}^{\prime(s)} \wedge \mathbf{g}_{\mathrm{I}}^{\prime(s)}\right\|} \geq \frac{\left\|\mathbf{g}_{\mathrm{I}}^{\prime(s)}\right\|}{\left\|\mathbf{g}_{\mathrm{III}}^{\prime(s)}\right\|}\left(\frac{\left\|\mathbf{g}_{\mathrm{I}}^{\prime(s)}\right\|}{\left\|\mathbf{g}_{\mathrm{III}}^{\prime(s)}\right\|}+2\right)
$$

i.e.:

$$
1+\frac{\left\|\mathbf{g}_{\mathrm{I}}^{(s)} \wedge \mathbf{g}_{\mathrm{II}}^{\prime(s)}\right\|}{\left\|\mathbf{g}_{\mathrm{III}}^{(s)} \wedge \mathbf{g}_{\mathrm{I}}^{\prime(s)}\right\|}+\frac{\left\|\mathbf{g}_{\mathrm{II}}^{\prime(s)} \wedge \mathbf{g}_{\mathrm{III}}^{(s)}\right\|}{\left\|\mathbf{g}_{\mathrm{III}}^{\prime(s)} \wedge \mathbf{g}_{\mathrm{I}}^{\prime(s)}\right\|} \leq \frac{\left\|\mathbf{g}_{\mathrm{III}}^{(s)}\right\|}{\left\|\mathbf{g}_{\mathrm{I}}^{\prime(s)}\right\|\left(\| \frac{\left\|\mathbf{g}_{\mathrm{I}}^{\prime(s)}\right\|}{\left\|\mathbf{g}_{\mathrm{II}}^{\prime(s)}\right\|}+2\right)}
$$

To obtain that, it's enough to show:

$$
1+\frac{\left\|\mathbf{g}_{\mathrm{I}}^{\prime(s)} \wedge \mathbf{g}_{\mathrm{II}}^{(s)}\right\|}{\left\|\mathbf{g}_{\mathrm{III}}^{\prime(s)} \wedge \mathbf{g}_{\mathrm{I}}^{\prime(s)}\right\|}+\frac{\left\|\mathbf{g}_{\mathrm{II}}^{\prime(s)} \wedge \mathbf{g}_{\mathrm{III}}^{(s)}\right\|}{\left\|\mathbf{g}_{\mathrm{III}}^{\prime(s)} \wedge \mathbf{g}_{\mathrm{I}}^{\prime(s)}\right\|} \leq \frac{\left\|\mathbf{g}_{\mathrm{III}}^{(s)}\right\|}{2.1\left\|\mathbf{g}_{\mathrm{I}}^{\prime(s)}\right\|},
$$

i.e.:

$$
\frac{2.1\left\|\mathbf{g}_{\mathrm{I}}^{\prime(s)}\right\|}{\left\|\mathbf{g}_{\text {III }}^{\prime(s)}\right\|}+\frac{2.1\left\|\mathbf{g}_{\mathrm{I}}^{\prime(s)}\right\|\left\|\mathbf{g}_{\mathrm{I}}^{\prime(s)} \wedge \mathbf{g}_{\mathrm{II}}^{\prime(s)}\right\|}{\left\|\mathbf{g}_{\text {III }}^{\prime(s)}\right\|\left\|\mathbf{g}_{\text {III }}^{\prime(s)} \wedge \mathbf{g}_{\mathrm{I}}^{\prime(s)}\right\|}+\frac{2.1\left\|\mathbf{g}_{\mathrm{I}}^{\prime(s)}\right\|\left\|\mathbf{g}_{\mathrm{II}}^{\prime(s)} \wedge \mathbf{g}_{\mathrm{III}}^{\prime(s)}\right\|}{\left\|\mathbf{g}_{\mathrm{III}}^{\prime(s)}\right\|\left\|\mathbf{g}_{\mathrm{III}}^{\prime(s)} \wedge \mathbf{g}_{\mathrm{I}}^{\prime(s)}\right\|} \leq 1
$$

We have:

$$
\begin{aligned}
\frac{2.1\left\|\mathbf{g}_{\mathrm{I}}^{\prime(s)}\right\|}{\left\|\mathbf{g}_{\mathrm{III}}^{\prime(s)}\right\|} \frac{\left\|\mathbf{g}_{\mathrm{I}}^{\prime(s)} \wedge \mathbf{g}_{\mathrm{II}}^{\prime(s)}\right\|}{\left\|\mathbf{g}_{\mathrm{III}}^{\prime(s)} \wedge \mathbf{g}_{\mathrm{I}}^{\prime(s)}\right\|} & =\frac{2.1\left\|\mathbf{g}_{\mathrm{I}}^{\prime(s)}\right\|}{\left\|\mathbf{g}_{\mathrm{III}}^{(s)}\right\|} \frac{\sin \left(\mid\left(\mathbf{g}_{\mathrm{I}}^{\prime(s)}, \mathbf{g}_{\mathrm{II}}^{\prime(s)}\right) \|\right)\left\|\mathbf{g}_{\mathrm{II}}^{(s)}\right\|}{\sin \left(\mid\left(\mathbf{g}_{\mathrm{I}}^{\prime(s)}, \mathbf{g}_{\mathrm{III}}^{\prime(s)}\right) \|\right)\left\|\mathbf{g}_{\mathrm{III}}^{\prime(s)}\right\|} \\
& \leq \frac{2.1 \times 0.1}{\sin \left(\frac{17 \pi}{31}\right)} \leq 0.22
\end{aligned}
$$


In addition:

$$
\begin{aligned}
\frac{2.1\left\|\mathbf{g}_{\mathrm{I}}^{\prime(s)}\right\|}{\left\|\mathbf{g}_{\text {III }}^{(s)}\right\|} \cdot \frac{\left\|\mathbf{g}_{\text {II }}^{\prime(s)} \wedge \mathbf{g}_{\text {III }}^{\prime(s)}\right\|}{\left\|\mathbf{g}_{\text {III }}^{(s)} \wedge \mathbf{g}_{\mathrm{I}}^{\prime(s)}\right\|} & =\frac{2.1 \sin \left(\left|\left(\mathbf{g}_{\text {III }}^{\prime(s)}, \mathbf{g}_{\text {II }}^{\prime(s)}\right)\right|\right)\left\|\mathbf{g}_{\text {II }}^{\prime(s)}\right\|}{\sin \left(\mid\left(\mathbf{g}_{\text {III }}^{\prime(s)}, \mathbf{g}_{\mathrm{I}}^{\prime(s)}\right) \|\right)\left\|\mathbf{g}_{\text {III }}^{\prime(s)}\right\|} \\
& \leq \frac{2.1 \sin \left(\frac{30 \pi}{31}\right)}{\sin \left(\frac{17 \pi}{31}\right)} \leq 0.22 .
\end{aligned}
$$

Finally:

$$
\frac{2.1 \times\left\|\mathbf{g}_{I}^{\prime(s)}\right\|}{\left\|\mathbf{g}_{\text {III }}^{\prime(s)}\right\|} \leq 2.1 \times 0.1 \leq 0.21
$$

The three last inequalities lead to the sufficient condition: (Ineq 4.2). Then we have proved:

$$
\frac{A^{(s)}}{\left\|\mathbf{g}_{\text {III }}^{\prime(s)}\right\|^{2}} \leq \frac{A^{(s+1)}}{\left\|\mathbf{g}_{\text {III }}^{(s+1)}\right\|^{2}}
$$

If $(s+1) \in T$, the proof of (Ineq 4.1) is finished. If now $(s+1) \notin T$, then we have both $\left\|\mathbf{g}_{\mathrm{III}}^{\prime(s+1)}\right\| \leq\left\|\mathbf{g}_{\mathrm{III}}^{(s+2)}\right\|$, and $A^{(s)} \leq A^{(s+2)}$ then $\frac{A^{(s)}}{\left\|\mathbf{g}_{\mathrm{III}}^{\prime(s)}\right\|^{2}} \leq$ $\frac{A^{(s+2)}}{\left\|\mathbf{g}_{I I I}^{\prime(s+2)}\right\|^{2}}$ and again the proof of (Ineq 4.1) is finished.

In the same way, as long as $(s+i) \notin T$, for $i=1,2, \ldots$, we have:

$$
\frac{A^{(s)}}{\left\|\mathbf{g}_{\text {III }}^{\prime(s)}\right\|^{2}} \leq \frac{A^{(s+2)}}{\left\|\mathbf{g}_{\text {III }}^{\prime(s+2)}\right\|^{2}} \leq \frac{A^{(s+3)}}{\left\|\mathbf{g}_{\text {III }}^{\prime(s+3)}\right\|^{2}} \leq \ldots \leq \frac{A^{(s+i)}}{\left\|\mathbf{g}_{\text {III }}^{\prime(s+i)}\right\|^{2}} \leq \ldots \leq \frac{A^{\left(s^{\prime}\right)}}{\left\|\mathbf{g}_{\text {III }}^{\prime\left(s^{\prime}\right)}\right\|^{2}},
$$

$s^{\prime}$ being the successor of $s$ in $T$. Then $\frac{A^{(s)}}{\left\|\mathbf{g}_{\text {III }}^{(s)}\right\|^{2}} \leq \frac{A^{\left(s^{\prime}\right)}}{\left\|\mathbf{g}_{\text {III }}^{\left(s^{\prime}\right)}\right\|^{2}}$ and the conclusion (Ineq 4.1) is reached in the case (II \I).

The reasoning is similar in all the four cases.

Then the conclusion of the Monotonic Sequence Lemma is established. So is the Geometrical Theorem, and also the Dirichlet Theorem and the Lagrange Theorem, but the last one only in a special case. We have to prove it generally.

\section{Lagrange Theorem from Dirichlet properties: complete demonstration}

Now, using the Theorem on Dirichlet Properties, we prove the Lagrange Theorem with the help of some Definitions, Lemma, Propositions. First we give the statements, then the proofs. 


\subsection{Definition and Statements of §5.}

Definition (Max-Dirichlet Property). It will be said that a sequence $\left(\mathbf{P}^{(s)}\right)=\left(\mathbf{p}_{0}^{(s)}, \mathbf{p}_{1}^{(s)}, \mathbf{p}_{2}^{(s)}\right)$ of triplets of integer vectors has the max-Dirichlet Property concerning $\mathbb{D}=\mathbb{R} \mathbf{X}$ (resp: $\mathbb{P}=\mathbf{X}^{\perp}$ ) if there exists an infinite subset $S$ of $\mathbb{N}$ such that: $\sup _{s \in S}\left[\left(\max _{i=0,1,2}\left\|\mathbf{p}_{i}^{\prime(s)}\right\|\right)^{2}\left(\max _{i=0,1,2}\left\|\mathbf{p}_{i}^{\prime \prime(s)}\right\|\right)\right]<+\infty$, with

$$
\lim _{s \rightarrow+\infty, s \in S}\left(\max _{i=0,1,2}\left\|\mathbf{p}_{i}^{\prime(s)}\right\|\right)=0\left(\operatorname{resp}: \lim _{s \rightarrow+\infty, s \in S}\left(\max _{i=0,1,2}\left\|\mathbf{p}_{i}^{\prime \prime(s)}\right\|\right)=0\right) .
$$

Lemma 16 (Polarity and Dirichlet Property). Let $\left(\mathbf{P}^{(s)}\right)$ be a sequence of integer matrices, all with the same determinant $D>0$, up to the sign, $i d$ est, for each $s \in \mathbb{N}$, $\operatorname{det}\left(\mathbf{P}^{(s)}\right)=\varepsilon^{(s)} D$, with $\varepsilon^{(s)} \in\{-1 ; 1\}$. Let $\left(\mathbf{P}^{(s)}\right)^{*}$ be the polar matrix of $\mathbf{P}^{(s)}$. Let $\mathbf{X}$ be a triplet of rationally independent real numbers.

If the sequence $\left(\mathbf{P}^{(s)}\right)$ has the max-Dirichlet Property for the plane $\mathbb{P}=$ $\mathbf{X}^{\perp}$ then the sequence $\left(D .\left(\mathbf{P}^{(s)}\right)^{*}\right)$ has the max-Dirichlet Property for the line $\mathbb{D}=\mathbb{R} \mathbf{X}$.

Proposition 1. Let $\theta$ be a real root of a third degree irreducible polynomial $P(t)=t^{3}-m t-n$, with $m$ and $n$ rationals. Let the vector $\boldsymbol{\Theta}$ be $\boldsymbol{\Theta}={ }^{\mathrm{T}}\left(1, \theta, \theta^{2}\right)$. Let $\mathbf{R}$ be any rational matrix, with $\operatorname{det}(\mathbf{R}) \neq 0$, such that $C \mathbf{R}$ has integral coefficients, with $C$ an integer. Let $\mathbf{X}$ be: $\mathbf{X}=\mathbf{R} \boldsymbol{\Theta}=$ $\mathbf{R}^{\mathrm{T}}\left(1, \theta, \theta^{2}\right)$. Let's suppose that: $\mathbf{X}={ }^{\mathrm{T}}\left(x_{0}, x_{1}, x_{2}\right)$, with $0<x_{0}<x_{1}<$ $x_{2}$. Let $\left(\mathbf{G}^{(s)}\right)$ be the sequence of integer matrices generated by the Smallest Vector Algorithm with initial value $\mathbf{X}=\mathbf{R} \Theta$.

Then the sequence $\left(\mathbf{G}^{(s)}\right)$ has the max-Dirichlet Property for the approximation of $\mathbb{P}=\mathbf{X}^{\perp}$ and $\left(C .{ }^{\mathrm{T}} \mathbf{R} \mathbf{G}^{(s)}\right)$ has the max-Dirichlet Property for the approximation of $\boldsymbol{\Pi}=\boldsymbol{\Theta}^{\perp}$. Moreover, there exists an integer $A$ such that the matrices $\mathbf{A}^{(s)}=A \mathbf{R}^{-1}\left(\mathbf{G}^{(s)}\right)^{*}$ are integer, and such that the sequence $\left(\mathbf{A}^{(s)}\right)$ has also the max-Dirichlet Property for the approximation of $\boldsymbol{\Delta}=\mathbb{R} \boldsymbol{\Theta}$.

Proposition 2. Let $\theta$ and $\boldsymbol{\Theta}$ be like in the previous Proposition. Let $\boldsymbol{\Delta}$ be: $\boldsymbol{\Delta}=\mathbb{R} \boldsymbol{\Theta}$. Let $\left(\mathbf{A}^{(s)}\right)$ be any sequence of integer matrices having the maxDirichlet Property for $\boldsymbol{\Delta}$, and all having the same determinant $D>0$, up to the sign. Let $\left(\mathbf{J}^{(s)}\right)$ be the polar matrices of the $\left(\mathbf{A}^{(s)}\right)$. Then there exists a sequence of rational matrices $\left(\mathbf{M}^{(s)}\right)$ and an integer $Q$, which depends only on $m$ and $n$, such that: 
- For each s, $\boldsymbol{\Theta}$ is an eigenvector for $\mathbf{M}^{(s)}$;

- $\liminf _{s \rightarrow+\infty}\left(\left\|D Q{ }^{\mathrm{T}} \mathbf{M}^{(s)} \mathbf{J}^{(s)}\right\|\right)<+\infty$, the matrices $\left(D Q{ }^{\mathrm{T}} \mathbf{M}^{(s)} \mathbf{J}^{(s)}\right)$ having integral coefficients.

Proposition 3. Let $\theta$ be, like in the two previous propositions, a real root of a third degree irreducible polynomial $P(t)=t^{3}-m t-n$, with $m$ and $n$ rationals. Let $\mathbf{X}=\mathrm{T}^{\mathrm{T}}\left(x_{0}, x_{1}, x_{2}\right)$ be a free triplet of three positive real numbers from the ring $\mathbb{Q}[\theta]$. Then the Smallest Vector Algorithm applied on $\mathbf{X}$ makes a loop: there exist integers $s$ and $t, s \neq t$, and a real number $\lambda$ such that:

$$
\left(\left\|\mathbf{g}_{0}^{\prime \prime(s)}\right\|,\left\|\mathbf{g}_{1}^{\prime \prime(s)}\right\|,\left\|\mathbf{g}_{2}^{\prime \prime(s)}\right\|\right)=\lambda\left(\left\|\mathbf{g}_{0}^{\prime \prime(t)}\right\|,\left\|\mathbf{g}_{1}^{\prime \prime(t)}\right\|,\left\|\mathbf{g}_{2}^{\prime \prime(t)}\right\|\right) .
$$

5.2. Demonstration of the Lemma. Let's denote:

$$
\left(\mathbf{P}^{(s)}\right)^{*}=\mathbf{Q}^{(s)}=\left(\mathbf{q}_{0}^{(s)}, \mathbf{q}_{1}^{(s)}, \mathbf{q}_{2}^{(s)}\right)
$$

then, for any direct circular permutation $(i, j, k)$ of $(0,1,2)$, forgetting the indices ${ }^{(s)}$ we have:

$$
\mathbf{q}_{i}=\frac{\varepsilon}{D}\left(\mathbf{p}_{j} \wedge \mathbf{p}_{k}\right) ; \mathbf{q}_{i}^{\prime}=\frac{\varepsilon}{D}\left(\mathbf{p}_{j}^{\prime \prime} \wedge \mathbf{p}_{k}^{\prime}+\mathbf{p}_{j}^{\prime} \wedge \mathbf{p}_{k}^{\prime \prime}\right) ; \mathbf{q}_{i}^{\prime \prime}=\frac{\varepsilon}{D}\left(\mathbf{p}_{j}^{\prime} \wedge \mathbf{p}_{k}^{\prime}\right) .
$$

Let's denote:

$$
\max _{i=0,1,2}\left\|\mathbf{p}_{i}^{\prime(s)}\right\|=\mu^{\prime(s)} ; \max _{i=0,1,2}\left\|\mathbf{p}_{i}^{\prime \prime(s)}\right\|=\mu^{\prime \prime(s)} .
$$

By hypothesis, $\left(\mu^{\prime(s)}\right)^{2} \mu^{\prime \prime(s)}<L$ holds for some $L$ and for every $s$ in the infinite set $S$. For each $i$, and any $s \in S$, we have: $\left\|\mathbf{q}_{i}^{\prime(s)}\right\| \leq \frac{2}{D} \mu^{\prime(s)} \mu^{\prime \prime(s)}$, and $\left\|\mathbf{q}_{i}^{\prime \prime(s)}\right\| \leq \frac{1}{D}\left(\mu^{\prime(s)}\right)^{2}$. Then

$$
\left(\max _{i=0,1,2}\left\|\mathbf{q}_{i}^{\prime(s)}\right\|\right)^{2}\left(\max _{i=0,1,2}\left\|\mathbf{q}_{i}^{\prime \prime(s)}\right\|\right) \leq \frac{4}{D^{3}}\left(\left(\mu^{\prime(s)}\right)^{2} \mu^{\prime \prime(s)}\right)^{2} \leq \frac{4 L^{2}}{D^{3}},
$$

and then:

$$
\left(\max _{i=0,1,2}\left\|D \mathbf{q}_{i}^{\prime(s)}\right\|\right)^{2}\left(\max _{i=0,1,2}\left\|D \mathbf{q}_{i}^{\prime \prime(s)}\right\|\right) \leq 4 L^{2}
$$

We have to prove in addition that the limit of the first factor is null. By hypothesis:

$$
\lim _{s \rightarrow+\infty, s \in S}\left(\max _{i=0,1,2}\left\|\mathbf{p}_{i}^{\prime \prime(s)}\right\|\right)=0
$$

This implies:

$$
\lim _{s \rightarrow+\infty, s \in S}\left(\max _{i=0,1,2}\left\|\mathbf{p}_{i}^{\prime(s)}\right\|\right)=+\infty
$$


Otherwise, the set of all the integer vectors $\mathbf{p}_{i}^{(s)}$, with $s$ in some infinite set $T \subset S$, would be bounded, and then finite. Then the sequence $\left(\max _{i=0,1,2}\left\|\mathbf{p}_{i}^{\prime \prime(s)}\right\|\right)_{s \in T}$ would have a non-null minimum. Contradiction!

Then we have: $\lim _{s \rightarrow+\infty, s \in S} \mu^{\prime(s)}=+\infty$. But we also have, for every $s \in S$,

$$
\left\|\mathbf{q}_{i}^{\prime(s)}\right\| \leq \frac{2}{D} \mu^{\prime(s)} \mu^{\prime \prime(s)}=\frac{2}{D} \frac{\left(\mu^{\prime(s)}\right)^{2} \mu^{\prime \prime(s)}}{\mu^{\prime(s)}} \leq \frac{2 L}{D} \frac{1}{\mu^{\prime(s)}} .
$$

Then $\lim _{s \rightarrow+\infty, s \in S}\left(\max _{i=0,1,2}\left\|D \mathbf{q}_{i}^{\prime(s)}\right\|\right)=0$. We have established that the sequence $\left(D \cdot\left(\mathbf{P}^{(s)}\right)^{*}\right)$ has the max Dirichlet Property for the line $\mathbb{D}=\mathbb{R} \mathbf{X}$.

5.3. Demonstration of Proposition 1. Let $\theta$ be a real root of a third degree irreducible polynomial $P(t)=t^{3}-m t-n$, where $m$ and $n$ are rationals.

For the initial value $\mathbf{X}=\mathbf{R} \Theta=\mathbf{R}^{\mathrm{T}}\left(1, \theta, \theta^{2}\right)$, let $\left(\mathbf{B}^{(s)}\right)$ and $\left(\mathbf{G}^{(s)}\right)$ be the sequences of integral matrices generated by the Smallest Vector Algorithm.

First, we establish that the couple $(\mathbb{P}, \mathbb{D})=\left(\mathbf{X}^{\perp}, \mathbb{R} \mathbf{X}\right)$ is badly approximable, in the sense of the Lemma 8 and the following Definition in Subsection 3.2.

By a classical theorem that we have already cited, (see [6] (Cassels), Theorem III, page 79 , statement $(2))$ the couple $\left(\boldsymbol{\Theta}^{\perp}, \mathbb{R} \boldsymbol{\Theta}\right)$ is badly approximable. Then:

$$
\inf _{\mathbf{k} \text { integer } \neq \mathbf{0}}\left[|\mathbf{k} \bullet \Theta| \cdot\|\mathbf{k}\|^{2}\right]>0
$$

Let's suppose that the couple $(\mathbb{P}, \mathbb{D})=\left(\mathbf{X}^{\perp}, \mathbb{R} \mathbf{X}\right)$ is NOT badly approximable. Then we would have:

$$
\inf _{\mathbf{h} \text { integer } \neq \mathbf{0}}\left[|\mathbf{h} \bullet \mathbf{R} \boldsymbol{\Theta}| \cdot\|\mathbf{h}\|^{2}\right]=0
$$

then

$$
\inf _{\mathbf{h} \text { integer } \neq \mathbf{0}}\left[\left|\left({ }^{\mathrm{T}} \mathbf{R h}\right) \bullet \boldsymbol{\Theta}\right| \cdot\left\|{ }^{\mathrm{T}} \mathbf{R h}\right\|^{2}\right]=0 .
$$

Let $q$ be an integer number such that $q \mathbf{R}$ has integral coefficient; then

$$
\inf _{\mathbf{h} \text { integer } \neq \mathbf{0}}\left[\left|\left(q^{\mathrm{T}} \mathbf{R h}\right) \bullet \boldsymbol{\Theta}\right| \cdot\left\|q^{\mathrm{T}} \mathbf{R h}\right\|^{2}\right]=0,
$$

with $\left(q^{\mathrm{T}} \mathbf{R h}\right)$ non-null integer. Then

$$
\inf _{\mathbf{k} \text { integer } \neq \mathbf{0}}\left[|\mathbf{k} \bullet \Theta| \cdot\|\mathbf{k}\|^{2}\right]=0 \text {. }
$$


Contradiction. Then the couple $(\mathbb{P}, \mathbb{D})=\left(\mathbf{X}^{\perp}, \mathbb{R} \mathbf{X}\right)$ is badly approximable.

By the Dirichlet Properties Theorem of Subsection 1.3., part b), the sequence $\left(\mathbf{G}^{(s)}\right)$, generated from $\mathbf{X}$ by the Smallest Vector Algorithm, have the max-Dirichlet Property for the approximation of $\mathbb{P}=\mathbf{X}^{\perp}$. There exists an infinite subset $S$ of $\mathbb{N}$ such that

$$
\sup _{s \in S}\left[\left(\max _{i=0,1,2}\left|\mathbf{g}_{i}^{(s)} \bullet \mathbf{R} \Theta\right|\right)\left(\max _{i=0,1,2}\left\|\mathbf{g}_{i}^{(s)}\right\|\right)^{2}\right]<+\infty,
$$

with $\lim _{s \rightarrow+\infty, s \in S}\left(\max _{i=0,1,2}\left|\mathbf{g}_{i}^{(s)} \bullet \mathbf{R \Theta}\right|\right)=0$. Then:

$$
\sup _{s \in S}\left[\left(\max _{i=0,1,2}\left|\left(C \cdot{ }^{\mathrm{T}} \mathbf{R g}_{i}^{(s)}\right) \bullet \Theta\right|\right)\left(\max _{i=0,1,2}\left\|C \cdot{ }^{\mathrm{T}} \mathbf{R g}_{i}^{(s)}\right\|\right)^{2}\right]<+\infty \text {, }
$$

with $\lim _{s \rightarrow+\infty, s \in S}\left(\max _{i=0,1,2}\left|\left(C \cdot{ }^{\mathrm{T}} \mathbf{R g}_{i}^{(s)}\right) \bullet \boldsymbol{\Theta}\right|\right)=0$. That means that the sequence $\left(C \cdot{ }^{\mathrm{T}} \mathbf{R} \mathrm{G}^{(s)}\right)$ has the max-Dirichlet Property for the approximation of $\boldsymbol{\Pi}=\boldsymbol{\Theta}^{\perp}$. But the $\left(C \cdot{ }^{\mathrm{T}} \mathbf{R} \mathbf{G}^{(s)}\right)$ have all the same determinant $\left(C^{3} \operatorname{det}(\mathbf{R})\right)$, up to the sign. Let $A$ be $A=C^{3} \operatorname{det}(\mathbf{R})$. Then, by the previous Lemma, the sequence $\left(A\left({ }^{\mathrm{T}} \mathbf{R} \mathbf{G}^{(s)}\right)^{*}\right)=\left(A \mathbf{R}^{-1}\left(\mathbf{G}^{(s)}\right)^{*}\right)$ of integer matrices has the max-Dirichlet Property for the approximation of $\boldsymbol{\Delta}=\mathbb{R} \boldsymbol{\Theta}$.

5.4. Demonstration of Proposition 2. Let $\left(\mathbf{A}^{(s)}\right)$ be a sequence of integer matrices having the max-Dirichlet Property for

$$
\boldsymbol{\Delta}=\mathbb{R} \boldsymbol{\Theta}=\mathbb{R}^{\mathrm{T}}\left(1, \theta, \theta^{2}\right),
$$

with $\theta^{3}=m \theta+n$.

We suppose that the matrices $\mathbf{A}^{(s)}$ have all the same determinant $D>0$, up to the sign, which means that for each $s \in \mathbb{N}$, $\operatorname{det}\left(\mathbf{A}^{(s)}\right)=\varepsilon^{(s)} D$, with $\varepsilon^{(s)} \in\{-1 ; 1\}$.

Let $\left(\mathbf{a}_{0}^{(s)}, \mathbf{a}_{1}^{(s)}, \mathbf{a}_{2}^{(s)}\right)$ be the column vectors of $\mathbf{A}^{(s)}$. We choose one of these three vectors, say $\mathbf{a}_{0}^{(s)}$, which will be more simply denoted: $\mathbf{a}^{(s)}:=\mathbf{a}_{0}^{(s)}$. Let's define its coordinates by: $\mathbf{a}^{(s)}=\mathrm{T}\left(a_{x}^{(s)}, a_{y}^{(s)}, a_{z}^{(s)}\right)$.

Let's denote: $\mu^{\prime(s)}=\max _{i=0,1,2}\left\|\mathbf{a}_{i}^{\prime(s)}\right\|$ and $\mu^{\prime \prime(s)}=\max _{i=0,1,2}\left\|\mathbf{a}_{i}^{\prime \prime(s)}\right\|$. We suppose that there exist an infinite set $S$ of integers and a real number $L$ such that for each $s \in S$, the inequality $\left(\mu^{\prime(s)}\right)^{2} \mu^{\prime \prime(s)}<L$ holds. Let $s$ be any element of $S$. From now on, we may omit the indices ${ }^{(s)}$. 
The notation $\mathbf{M}=\mathbf{M}^{(s)}$ will denote the rational matrix

$$
\mathbf{M}^{(s)}=\left(\begin{array}{ccc}
-m \cdot a_{x}+a_{z} & a_{y} & a_{x} \\
n \cdot a_{x} & a_{z} & a_{y} \\
n \cdot a_{y} & n \cdot a_{x}+m \cdot a_{y} & a_{z}
\end{array}\right) .
$$

If $Q$ is a natural such that $Q m$ and $Q n$ are integers, then $Q \mathbf{M}^{(s)}$ has integral coefficients.

We have:

$$
\mathbf{M} \Theta=\mathbf{M}\left(\begin{array}{l}
1 \\
\theta \\
\theta^{2}
\end{array}\right)=\left(-m \cdot a_{x}+a_{z}+a_{y} \theta+a_{x} \theta^{2}\right)\left(\begin{array}{l}
1 \\
\theta \\
\theta^{2}
\end{array}\right) .
$$

Let's denote by $\lambda$ the following element of $\mathbb{Z}[\theta]$ :

$$
\lambda:=\left(-m \cdot a_{x}+a_{z}+a_{y} \theta+a_{x} \theta^{2}\right) .
$$

Then we have $\mathbf{M} \boldsymbol{\Theta}=\lambda \boldsymbol{\Theta} ; \lambda$ is an eigenvalue of $\mathbf{M}$ with eigenvector $\boldsymbol{\Theta}$.

Let $\left(\mathbf{J}^{(s)}\right)$ the polar matrices of the $\left(\mathbf{A}^{(s)}\right)$. We consider the sequence of the matrices $\boldsymbol{\Pi}^{(s)}=\mathbf{\Pi}={ }^{\mathrm{T}} \mathbf{M J}$.

Let $\left(\mathbf{a}^{\# \#}, \mathbf{a}^{\#}, \mathbf{a}\right)$ be the three column vectors of $\mathbf{M}$. Then, with scalar products:

$$
\boldsymbol{\Pi}={ }^{\mathrm{T}} \mathbf{M J}=\left(\begin{array}{ccc}
\mathbf{a}^{\# \#} \bullet \mathbf{j}_{0} & \mathbf{a}^{\# \#} \bullet \mathbf{j}_{1} & \mathbf{a}^{\# \#} \bullet \mathbf{j}_{2} \\
\mathbf{a}^{\#} \bullet \mathbf{j}_{0} & \mathbf{a}^{\#} \bullet \mathbf{j}_{1} & \mathbf{a}^{\#} \bullet \mathbf{j}_{2} \\
\mathbf{a} \bullet \mathbf{j}_{0} & \mathbf{a} \bullet \mathbf{j}_{1} & \mathbf{a} \bullet \mathbf{j}_{1}
\end{array}\right)=\left(\pi_{i, j}\right)
$$

say, with $i=1,2,3 ; j=1,2,3$.

We now have to find an upper bound for each of the $\left|\pi_{i, j}\right|$.

We have:

$$
\mathbf{a}^{\#}=\mathbf{Q}^{\#}\left(\begin{array}{c}
a_{x} \\
a_{y} \\
a_{z}
\end{array}\right)=\mathbf{Q}^{\#} \mathbf{a}
$$

with $\mathbf{Q}^{\#}=\left(\begin{array}{ccc}0 & 1 & 0 \\ 0 & 0 & 1 \\ n & m & 0\end{array}\right)$ and:

$$
\mathbf{a}^{\# \#}=\mathbf{Q}^{\# \#}\left(\begin{array}{c}
a_{x} \\
a_{y} \\
a_{z}
\end{array}\right)=\mathbf{Q}^{\# \#} \mathbf{a}
$$

with $\mathbf{Q}^{\# \#}=\left(\begin{array}{ccc}-m & 0 & 1 \\ n & 0 & 0 \\ 0 & n & 0\end{array}\right)$.

We have:

$$
\mathbf{Q}^{\#} \boldsymbol{\Theta}=\theta \mathbf{\Theta}, \text { and } \mathbf{Q}^{\# \#} \mathbf{\Theta}=\left(-m+\theta^{2}\right) \mathbf{\Theta}
$$


First let's consider the $\left|\mathbf{a}^{\#} \bullet \mathbf{j}_{i}\right|$.

Let $\nu$ be: $\nu:=\frac{\boldsymbol{\Theta}}{\|\boldsymbol{\Theta}\|}$. Then also $\mathbf{Q}^{\#} \nu=\theta \nu$.

With always the same kind of notations, we have $\mathbf{a}^{\#}=\mathbf{a}^{\# \prime}+\mathbf{a}^{\# \prime \prime}$, and: $\mathbf{a}^{\#} \bullet \mathbf{j}_{i}=\left(\mathbf{a}^{\# \prime}+\mathbf{a}^{\# \prime \prime}\right) \bullet\left(\mathbf{j}_{i}^{\prime}+\mathbf{j}_{i}^{\prime \prime}\right)=\mathbf{a}^{\# \prime} \bullet \mathbf{j}_{i}^{\prime}+\mathbf{a}^{\# \prime \prime} \bullet \mathbf{j}_{i}^{\prime \prime}=\mathbf{a}^{\# \prime} \bullet \mathbf{j}_{i}^{\prime}+\mathbf{a}^{\# \prime \prime} \bullet \mathbf{j}_{i}^{\prime \prime}$, with

$$
\mathbf{j}_{i}=\frac{\varepsilon}{D}\left(\mathbf{a}_{j} \wedge \mathbf{a}_{k}\right) ; \mathbf{j}_{i}^{\prime}=\frac{\varepsilon}{D}\left(\mathbf{a}_{j}^{\prime \prime} \wedge \mathbf{a}_{k}^{\prime}+\mathbf{a}_{j}^{\prime} \wedge \mathbf{a}_{k}^{\prime \prime}\right) ; \mathbf{j}_{i}^{\prime \prime}=\frac{\varepsilon}{D}\left(\mathbf{a}_{j}^{\prime} \wedge \mathbf{a}_{k}^{\prime} .\right) .
$$

Then:

$$
\left|\mathbf{a}^{\#} \bullet \mathbf{j}_{i}\right| \leq \frac{1}{D}\left(\left\|\mathbf{a}^{\# \prime}\right\| \mu^{\prime \prime} \mu^{\prime}++\left\|\mathbf{a}^{\# \prime}\right\| \mu^{\prime} \mu^{\prime \prime}+\left\|\mathbf{a}^{\# \prime \prime}\right\|\left(\mu^{\prime}\right)^{2}\right)
$$

and we have also $\mathbf{a}^{\#}=\mathbf{Q}^{\#} \mathbf{a}=\mathbf{Q}^{\#}\left(\mathbf{a}_{0}^{\prime}+\left\|\mathbf{a}_{0}^{\prime \prime}\right\| \nu\right)=\mathbf{Q}^{\#} \mathbf{a}_{0}^{\prime}+\left\|\mathbf{a}_{0}^{\prime \prime}\right\| \theta \nu$.

This proves first that the distance $\left\|\mathbf{a}^{\# \prime}\right\|$ between $\mathbf{a}^{\#}$ and $\mathbb{D}$ is less than $\left\|\mathbf{Q}^{\#} \mathbf{a}_{0}^{\prime}\right\|$ :

$$
\left\|\mathbf{a}^{\# \prime}\right\| \leq\left\|\mathbf{Q}^{\#} \mathbf{a}_{0}^{\prime}\right\| \leq\left\|\mathbf{Q}^{\#}\right\| \times\left\|\mathbf{a}_{0}^{\prime}\right\| \leq\left\|\mathbf{Q}^{\#}\right\| \mu^{\prime}
$$

(this using the norm of the matrix).

Moreover, we have $\mathbf{a}^{\# \prime}+\mathbf{a}^{\# \prime \prime}=\mathbf{a}^{\#}=\mathbf{Q}^{\#} \mathbf{a}_{0}^{\prime}+\left\|\mathbf{a}_{0}^{\prime \prime}\right\| \theta \nu$, then:

$$
\mathbf{a}^{\# \prime \prime}=\left\|\mathbf{a}_{0}^{\prime \prime}\right\| \theta \nu+\mathbf{Q}^{\#} \mathbf{a}_{0}^{\prime}-\mathbf{a}^{\# \prime} .
$$

Then:

$$
\left\|\mathbf{a}^{\# \prime \prime}\right\| \leq\left\|\mathbf{a}_{0}^{\prime \prime}\right\| \theta+2\left\|\mathbf{Q}^{\#}\right\| \times\left\|\mathbf{a}_{0}^{\prime}\right\| \leq \mu^{\prime \prime} \theta+2\left\|\mathbf{Q}^{\#}\right\| \mu^{\prime}
$$

Putting 5.2 and 5.3 in 5.1, we obtain:

$$
\left|\mathbf{a}^{\#(s)} \bullet \mathbf{j}_{i}^{(s)}\right| \leq \frac{1}{D}\left(\mu^{\prime(s)}\right)^{2} \mu^{\prime \prime(s)}\left(2\left\|\mathbf{Q}^{\#}\right\|+\theta\right)+\frac{2}{D}\left\|\mathbf{Q}^{\#}\right\|\left(\mu^{\prime(s)}\right)^{3},
$$

and then:

$$
\left|\mathbf{a}^{\#(s)} \bullet \mathbf{j}_{i}^{(s)}\right| \leq \frac{L}{D}\left(2\left\|\mathbf{Q}^{\#}\right\|+\theta\right)+\frac{2}{D}\left\|\mathbf{Q}^{\#}\right\|\left(\mu^{\prime(s)}\right)^{3} .
$$

The limit of the last term is 0 .

Then the set of the $\left|\mathbf{a}^{\#(s)} \bullet \mathbf{j}_{i}^{(s)}\right|$, with $s$ in $S$, is bounded. A similar demonstration shows that the $\left|\mathbf{a}^{\# \#(s)} \bullet \mathbf{j}_{i}^{(s)}\right|$ are also bounded and so are in an obvious way the $\left|\mathbf{a}_{0}^{(s)} \bullet \mathbf{j}_{i}^{(s)}\right|$. Then the set of the $\boldsymbol{\Pi}^{(s)}={ }^{\mathrm{T}} \mathbf{M}^{(s)} \mathbf{J}^{(s)}$ is bounded. But $\mathbf{J}^{(s)}=\left(\mathbf{A}^{(s)}\right)^{*}$, with $\operatorname{det}\left(\mathbf{A}^{(s)}\right)= \pm D$. Then the matrices $D \mathbf{J}^{(s)}$ have integral coefficients. We have seen that the matrices $Q \mathbf{M}^{(s)}$ have also integral coefficients. In addition, the sequence $\left(D Q{ }^{\mathrm{T}} \mathbf{M}^{(s)} \mathbf{J}^{(s)}\right)$ is bounded, and the proof is done. 
5.5. Demonstration of Proposition 3. Let $\theta$ be a real root of a third degree irreducible polynomial $P(t)=t^{3}-m t-n$, with $m$ and $n$ rationals. Let $\mathbf{X}={ }^{\mathrm{T}}\left(x_{0}, x_{1}, x_{2}\right)$ be a free triple of three positive real numbers from the ring $\mathbb{Q}[\theta]$. Let $\boldsymbol{\Theta}$ be $\boldsymbol{\Theta}={ }^{\mathrm{T}}\left(1, \theta, \theta^{2}\right)$. Then there exists a rational matrix $\mathbf{R}$, with $\operatorname{det}(\mathbf{R}) \neq 0$, such that $\mathbf{X}=\mathbf{R} \Theta$.

Then, by Proposition 1, there exists an integer $A$ such that the matrices $\mathbf{A}^{(s)}=A \mathbf{R}^{-1}\left(\mathbf{G}^{(s)}\right)^{*}$ are integer, and such that the sequence $\left(\mathbf{A}^{(s)}\right)$ has the max-Dirichlet Property for the approximations of $\boldsymbol{\Delta}=\mathbb{R} \Theta$. All the matrices $\mathbf{A}^{(s)}$ have the same determinant, say $D>0$, up to the sign; then, by Proposition 2, there exists a sequence of integer matrices $\left(\mathbf{M}^{(s)}\right)$ such that, for each $s, \boldsymbol{\Theta}$ is an eigenvector for $\mathbf{M}^{(s)}$ and $\liminf _{s \rightarrow+\infty}\left(\left\|D Q \cdot{ }^{\mathrm{T}} \mathbf{M}^{(s)} \mathbf{J}^{(s)}\right\|\right)<$ $+\infty$, the matrices $\left(D Q \cdot{ }^{\mathrm{T}} \mathbf{M}^{(s)} \mathbf{J}^{(s)}\right)$ having integral coefficients, with

$$
\mathbf{J}^{(s)}=\left(\mathbf{A}^{(s)}\right)^{*}=\left(A \mathbf{R}^{-1}\left(\mathbf{G}^{(s)}\right)^{*}\right)^{*}=A^{-1}{ }^{\mathrm{T}} \mathbf{R} \mathbf{G}^{(s)} .
$$

There exists an infinite subset $S$ of $\mathbb{N}$, such that the set of all the integer matrices $\left(D Q{ }^{\mathrm{T}} \mathbf{M}^{(s)} \mathbf{J}^{(s)}\right)$ with $s$ in $S$ is bounded; then it is finite. Then there exist $s$ and $t, t \neq s$, such that ${ }^{\mathrm{T}} \mathbf{M}^{(s) \mathrm{T}} \mathbf{R} \mathbf{G}^{(s)}={ }^{\mathrm{T}} \mathbf{M}^{(t) \mathrm{T}} \mathbf{R} \mathbf{G}^{(t)}$. By transposition: $\left(\mathbf{B}^{(s)}\right)^{-1} \mathbf{R} \mathbf{M}^{(s)}=\left(\mathbf{B}^{(t)}\right)^{-1} \mathbf{R} \mathbf{M}^{(t)}$. We apply that to the column vector $\boldsymbol{\Theta}$ : $\left(\mathbf{B}^{(s)}\right)^{-1} \mathbf{R} \mathbf{M}^{(s)} \boldsymbol{\Theta}=\left(\mathbf{B}^{(t)}\right)^{-1} \mathbf{R} \mathbf{M}^{(t)} \boldsymbol{\Theta}$; then, by "eigenvector", and because $\mathbf{R} \Theta=\mathbf{X}$, we have $\lambda^{(s)}\left(\mathbf{B}^{(s)}\right)^{-1} \mathbf{X}=\lambda^{(t)}\left(\mathbf{B}^{(t)}\right)^{-1} \mathbf{X}$, then $\left(\mathbf{B}^{(s)}\right)^{-1} \mathbf{X}=\frac{\lambda^{(t)}}{\lambda^{(s)}}\left(\mathbf{B}^{(t)}\right)^{-1} \mathbf{X}$.

This reads: $\mathbf{X}^{(s)}=\lambda \mathbf{X}^{(t)}$, with $\lambda=\frac{\lambda^{(t)}}{\lambda^{(s)}}$, and our Lagrange Theorem is proved if $\mathbf{X}=\mathrm{T}^{\mathrm{T}}\left(x_{0}, x_{1}, x_{2}\right)$ is a free triplet of three positive real numbers from the ring $\mathbb{Q}[\theta], \theta$ being a real root of a third degree irreducible polynomial $P(t)=t^{3}-m t-n$, with $m$ and $n$ rationals. Of course this case is general, as we're going to verify it.

5.6. From Proposition 3 to the Lagrange Theorem. This part is very quick. Let $\rho$ be a real root of a third degree irreducible polynomial $S(t)=t^{3}-a t^{2}-b t-c$, with with $a, b, c$ rationals. Then $\theta=\rho-\frac{a}{3}$ is a real root of a third degree irreducible polynomial $P(t)=t^{3}-m t-n$, with $m$ and $n$ rationals. If $x_{0}, x_{1}, x_{2}$ are elements of $\mathbb{Q}[\rho]$, they also belong to $\mathbb{Q}[\theta]=\mathbb{Q}[\rho]$. Then Proposition 3 implies the conclusion of the main Lagrange Theorem (first part). The second part of the theorem has been established in Section 2. 


\section{Bibliography and Themes related to this Paper}

The work nearest to the present paper is the book by A.J. Brentjes [3]. A lot of themes are in common: the approach of the continued fractions with matrices and linear algebra, the fact that non vectorial algorithms are used, the study of angular properties and of the needling phenomenon...Brentjes' book is mainly concerned with algebraic results, best approximation, and (strong) convergence, rather than with "Dirichlet" approximation, with the optimal exponent, or "Lagrange" results. However, it contains a Lagrangetype statement, in the Corollary, page 106, but with a lattice which is not $\mathbb{Z}^{3}$.

Most multidimensional continued fractions algorithms, among those which are additive (or subtractive, or multiplicative), are of the vectorial type. This means that in such an algorithm, the vector $\mathbf{X}^{(s+1)}$ depends only on $\mathbf{X}^{(s)}=\|\mathbf{X}\|\left(\mathbf{g}_{0}^{\prime \prime(s)}, \mathbf{g}_{1}^{\prime \prime(s)}, \mathbf{g}_{2}^{\prime \prime(s)}\right)$, in a simple way, and not on $\left(\mathbf{g}_{0}^{\prime(s)}, \mathbf{g}_{1}^{\prime(s)}, \mathbf{g}_{2}^{\prime(s)}\right)$ in the plane $\mathbb{P}$. In this case, the algorithm defines clearly a discrete dynamical system, the orbits of which are the sequences $\left(\mathbf{X}^{(s)}\right)$. There are a lot of interesting studies of these dynamical systems, by Fritz Schweiger, J.C. Lagarias and many others, but our algorithm, like Brentjes' one, is non vectorial, and different techniques are used. With such non vectorial algorithms, results everywhere may be obtained. With vectorial ones, most of the results are obtained almost everywhere.

Apart from Brentjes' book, there is another treatise by Fritz Schweiger on multidimensional continued fractions [22]. It is very complete and presents the general Brentjes' algorithms, but deals mainly with vectorial algorithms and dynamical systems.

The continued fractions are only a tool in the theory of Diophantine Approximation. Here we use two theorems by Minkowski in Geometry of Numbers. The references in these fields are for instance: [5], [6], [14], and [21].

In the area of algorithms which aim to best approximation, apart from the specific Brentjes' algorithm, we may cite the Furtwängler's algorithm [12] (an error was pointed out by K.M. Briggs, see his paper), which inspired Keith Briggs [4] and Vaughan Clarkson [9]; see also the Ph. D. thesis of V. Clarkson: [8].

There are some studies of the matrices of best approximations, which could be connected to our work: By J.C. Lagarias: [18], and [17], and a review by N.G. Moshchevitin: [20].

In the present paper, the result on best approximations is the Prism Lemma, at the beginning of Section 3. It is an easy result, but perhaps it clarifies the problem. It is more efficient if the hexagon it involves is balanced, and we have some results in this direction in this paper. 
J.C. Lagarias has also build in [19] a very interesting algorithm, which is additive but not positive, and which provides best approximations. See also the very complete paper by N. Chevallier: [7].

The LLL algorithm (named after A.K. Lenstra, H.W. Lenstra, L. Lovàsz) is very efficient in Number Theory. It provides good approximations, and even Dirichlet approximations, with the optimal exponent: see [2], by W. Bosma and I. Smeets. But maybe it is not designed to possess approximation properties with triplets of integer vectors, nor Lagrange properties, as the Smallest Vector Algorithm does.

There is an another kind of Multidimensional Continued Fractions, very different from the additive (i.e. subtractive) ones we have considered until now. These other constructions use stars of sails, obtained from hyperplanes and pyramids in $\mathbb{R}^{k}$. The original idea is due to K. Klein, H. Minkowski, and G. F. Voronoi. V. I. Arnold renewed the interest toward this theory: [1]. "Lagrange" results seem to have been obtained, by G. Lachaud, [16], E. Korkina [15], or O.N. German and E.L Lakshtanov: [13]. But their statements don't seem as simple as the Theorem 1 of the present work. In the cited paper, V.I. Arnold has written:

"The attempts to generalize to higher dimensions the algorithm (emphasized by V.I. Arnold) of continued fractions lead to complicate and ugly theories. For instance the sail corresponding to a cubical irrational number is a double-periodic surface. However the algorithms define instead of this surface a path on it. [...] the path is not periodic at all and looks like a rather chaotic object; it is unclear how to describe the cubic irrationals in terms of the combinatorics of this path".

We can just hope that Arnold was only partly right. It would be interesting to study the relation between the regularities we have pointed out in the "chaotic" paths generated by our algorithm for cubic numbers, and the symmetries of the corresponding sails.

\section{Acknowledgments}

I want to thank Professor Fritz Schweiger for his generous mathematical help, his encouragements, his numerous and accurate readings of my papers and his remarks. I also owe my gratitude to Professor Eugène Dubois, who has given a lot of his time to read a previous version of this paper and to Professor Michel Mendès-France, in Bordeaux I University. I also thank the anonymous referee, for having pointed out several gaps and mistakes in previous versions of some demonstrations. A friendly thank to my Dax colleague Philippe Paya, for all the fruitful talks and interesting remarks. 


\section{References}

[1] V.I. ARnold, Higher dimensional continued fractions. Regular and Chaotic Dynamics 3 (1998), $\mathbf{n}^{\circ} \mathbf{3}, 10-17$.

[2] W. Bosma and I. Smeets, An algorithm for finding approximations with optimal Dirichlet quality (http://arxiv.org/abs/1001.4455). Submitted.

[3] A.J. BrentJes, Multi-dimensional continued fraction algorithms. Mathematics Center Tracts 145, Mathematisch Centrum, Amsterdam, 1981.

[4] K.M. BRIGGS, On the Furtwängler algorithm for simultaneous rational approximation. Exp. Math. (to be submitted), 2001.

[5] J.W.S. CASsels, An Introduction to the Geometry of Numbers. Springer.

[6] J.W.S. CAssels, An Introduction to diophantine approximation. Cambridge University Press, 1957.

[7] N. Chevallier, Best Simultaneous Diophantine Approximations and Multidimensional Continued Fraction Expansions. Moscow J. of Combinatorics and Number Theory 3 (2013), $\mathbf{n}^{\circ} \mathbf{1}, 3-56$.

[8] I.V.L. Clarkson, Approximation of Linear Forms by Lattice Points, with applications to signal processing. PhD thesis, Australian National University, 1997.

[9] V. Clarkson, J. Perkins, and I. Mareels, An algorithm for best approximation of a line by lattice points in three dimensions. Technical report, 1995. 3rd Conference on Computational Algebra and Number Theory (CANT 95). Formerly online at wwwcrasys.anu.edu.au/Projects/pulseTrain/Papers/CPM95.ps.gz.

[10] H. Davenport, On a theorem of Furtwängler. J. London Math. Soc. 30 (1955), 186-195.

[11] H. Davenport, Simultaneous diophantine approximation. Proc. London Math. Soc. 2 (1952), 403-416.

[12] Ph. Furtwängler, Über die simultane Approximation von Irrationalzahlen I and II. Math. Annalen 96 (1927), 169-175 and Math. Annalen 99 (1928), 71-83.

[13] O.N. German And E.L LaKshtanov, On a multidimensional generalization of Lagrange's theorem on continued fractions. Izv. Math. 72:1 (2008), 47-61.

[14] J.F. Koksma, Diophantische Approximationen. Ergebnisse der Mathematik und ihrer Grenzgebiete 4 (1936), 409-571; and Chelsea Publishing Company, Amsterdam, 1982.

[15] E Korkina, La périodicité des fractions continues multidimensionnelles, C. R. Acad. Sci. Paris t.319, Série I (1994), 777-780.

[16] G. Lachaud, Polyèdre d'Arnol'd et voile d'un cône simplicial: analogues du théorème de Lagrange. C. R. Acad. Sci. Paris, t. 317, Série I (1993), 711-716.

[17] J.C. Lagarias, Best simultaneous diophantine approximations I. Growth rates of best approximation denominators. Trans. Am. Math. Soc. 272 (1980), 545-554.

[18] J.C. Lagarias, Best simultaneous diophantine approximations II. Behavior of consecutive best approximations. Pacific J. Math. 102, $\mathbf{n}^{\circ} \mathbf{1}$ (1982), 61-88.

[19] J.C. Lagarias, Geodesic multidimensional continued fractions, Proc. London Math. Soc, (3) (1994), 69, 231-244.

[20] N.G. Moshchevitin, Continued fractions, multidimensional Diophantine approximations and applications. J. de Théorie des Nombres de Bordeaux 11 (1999), 425-438.

[21] W. Schmidt, Diophantine approximation. Lectures Notes in Mathematics 785, Springer, 1980.

[22] F. SchweIger, Multidimensional Continued Fractions Algorithms. Oxford University Press, 2000 .

[23] F. SchweIGer, Was leisten mehrdimensionale Kettenbrüche. Mathematische Semesterberichte 53 (2006), 231-244.

Christian Drouin

26 Avenue d'Yreye

40510 SEIGNOSSE FRANCE

E-mail: christian.drouin@wanadoo.fr 